\title{
A study of the phase transition in 4D pure compact U(1) LGT on toroidal and spherical lattices
}

\author{
Isabel Campos ${ }^{1}$, Andrés Cruz ${ }^{2}$, Alfonso Tarancón ${ }^{3}$ \\ Departamento de Física Teórica, Facultad de Ciencias, Universidad de Zaragoza, 50009 Zaragoza, Spain
}

Received 10 March 1998; accepted 29 May 1998

\begin{abstract}
We have performed a systematic study of the phase transition in the pure compact $U(1)$ lattice gauge theory in the extended coupling parameter space $(\beta, \gamma)$ on toroidal and spherical lattices. The observation of a non-zero latent heat in both topologies for all investigated $\gamma \in[+0.2,-0.4]$, together with an exponent $\nu_{\text {eff }} \sim 1 / d$ when large enough lattices are considered, lead us to conclude that the phase transition is first order. For negative $\gamma$, our results point to an increasingly weak first-order transition as $\gamma$ is made more negative. (C) 1998 Elsevier Science B.V.
\end{abstract}

PACS: 11.15.Ha; 64.60.Fr; 05.70.Fh; 05.70.Jk

Keywords: Transient effects in phase transitions; Critical exponents; Thermodynamic properties of gauge theories

\section{Introduction and overview}

In this work the $4 D$ pure compact $\mathrm{U}(1)$ gauge theory is revisited. This model, being the prototype of gauge abelian interaction, has received a lot of attention through the years, since it constitutes a preliminary step in approaching QED. However, the questions related with the mechanisms driving the transition and with the order of the phase transition itself, have turned out to be a more controversial issue than one might expect from its apparent simplicity.

Through this section we shall summarize the main results obtained up to date.

\footnotetext{
${ }^{1}$ E-mail: isabel@sol.unizar.es

${ }^{2}$ E-mail: tarancon@sol.unizar.es

${ }^{3}$ E-mail: cruz@sol.unizar.es
} 
The $4 D$ pure compact $U(1)$ theory is known to undergo a phase transition between a region in which the photons are confined (strong coupling) and another region in which they carry a Coulomb potential (weak coupling). The theory can be discretized in the lattice using the Wilson action

$$
S_{\mathrm{W}}=\beta \sum_{\mathrm{P}}\left[1-\operatorname{Re} \operatorname{tr} U_{\mathrm{P}}\right] .
$$

Most of the work has been done on the hypercubic lattice with periodic boundary conditions, namely on the hypertorus. On this topology the pioneer studies (see e.g. Refs. [1-3]) pointed to a continuous transition since numerical simulations did not evidence signals of metastability. The confinement-deconfinement transition sets in at a coupling $\beta \sim 1$. The largest lattice size simulated in those works was $L=5$. However, as simulations in larger lattices became available to computer resources, metastabilities were evidenced, and two-peaked energy histograms could be observed from $L=6$ on (see e.g. Refs. [4-6]) pointing to a first-order character for the phase transition. This idea was also supported by several works that approached the problem from the point of view of the renormalization group [7-10].

Nowadays, though the general belief is that the transition is first order, there are still some puzzling questions. Namely, the numerical simulations up to $L=12$ do not reveal a stable latent heat, it is rather decreasing with the lattice size, and also, the associated critical exponent $\nu$ is always in the interval $\nu=0.29-0.32$, which is different from the first-order value $(\nu=0.25)$ and from the trivial second-order one, namely $\nu=0.5$. These facts led some authors to wonder about the possibility of a slowly vanishing latent heat, which could eventually go to zero in the thermodynamic limit. In short, the $4 D$ pure compact $U(1)$ theory could be critical provided the two peaks appearing in the simulations are a finite size effect. Due to the physical implications of a critical theory with non-trivial critical exponents this possibility was not completely ruled out.

Now, we go back in time to introduce the extended Wilson action. Besides the Wilson action, an action including a term proportional to the square of the plaquettes was proposed by Bhanot at the beginning of the eighties [11],

$$
S_{\mathrm{EW}}=\beta \sum_{\mathrm{p}}\left[1-\operatorname{Re} \operatorname{tr} U_{\mathrm{p}}\right]+\gamma \sum_{\mathrm{p}}\left[1-\operatorname{Re} \operatorname{tr} U_{\mathrm{p}}^{2}\right] .
$$

In that work the extended $(\beta, \gamma)$ parameter space was explored in $L=4,5$, and it was suggested that the phase transition line $\beta_{\mathrm{c}}(\gamma)$ became first order for $\gamma \geqslant+0.2$, while for $\gamma<0.2$ the transition appeared to be second order on those small lattices. The phase diagram is represented in Fig. 1.

Simulations in larger hypercubic lattices have shown that two metastable states are present for all, up to date, positive and negative simulated $\gamma$ values. What holds true is the observation that a positive $\gamma$ value reinforces the transition towards the first order, while a negative $\gamma$ value weakens the transition. In fact, the latent heat, $C_{\text {lat }}$, decreases with $\gamma$, and then, a coupling where $C_{\text {lat }}$ goes to zero was supposed to exist at some finite (negative) value of $\gamma$. This behavior induced some authors to conjecture about 


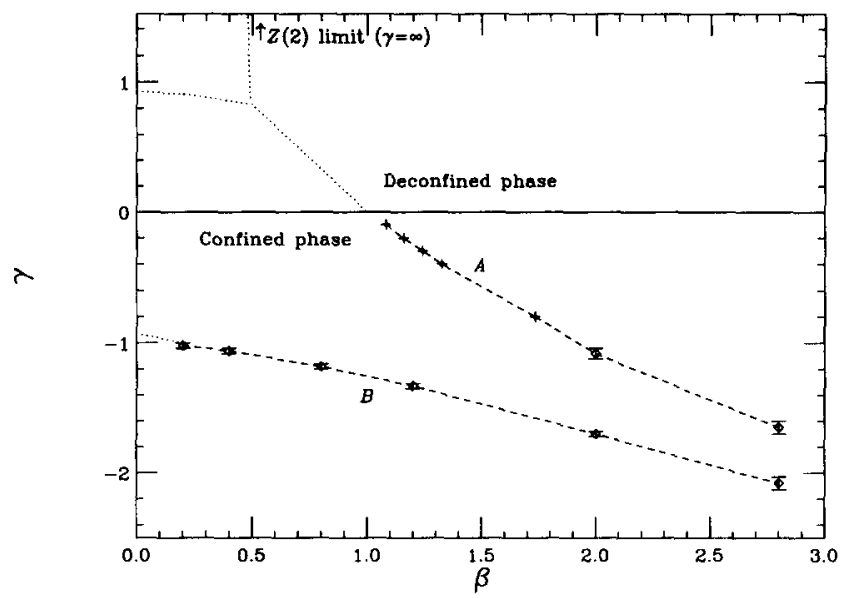

Fig. 1. Phase diagram of $U(1)$ in the extended $(\beta, \gamma)$ parameter space. The dotted lines have been taken from Ref. [11]. The symbols correspond to our simulations on the torus. The crosses correspond to the peak of $C_{\mathrm{V}}(L=16)$, the errors are not visible in this scale. The diamonds have been obtained with hysteresis cycles in $L=8$.

the existence of a tricritical point (TCP) at some negative coupling $\gamma_{\mathrm{TCP}}$ where the order of the phase transition could change from being first order to be continuous [12]. However, every simulation performed at negative $\gamma$ has exhibited double peak structures for large enough $L$. As for the Wilson action $(\gamma=0)$, for negative $\gamma$ a non-stable latent heat is revealed by simulating in lattice sizes in the range $L=4,12$.

From the point of view of the Renormalization Group this problem was studied in [13] and the results pointed to a phase transition line $\beta_{\mathrm{c}}(\gamma)$ of first order for all $\gamma$ except at the end, $\gamma=-\infty$, where the transition is second order with trivial exponents.

Nowadays, it is generally accepted that the transition is first order for positive $\gamma$ values. However, for $\gamma \leqslant 0$ two scenarios arise: (1) The phase transition line $\beta_{\mathrm{c}}(\gamma)$ is first order everywhere except at $\gamma=-\infty$; (2) There is a finite $\gamma_{\mathrm{TCP}} \leqslant 0$ from which on, the phase transition is second order.

As we have already remarked, all the previous discussions refer to simulations performed on the hypertorus.

Recently some authors have cast a new hue on this problem. It was suggested some years ago that the jump in the energy observed when the confining-deconfining transition takes place, could be due to the existence of closed monopole loops wrapping around the toroidal lattice, which due to the particular topology cannot be contracted to a point [14]. This hypothesis led authors to work on lattices homotopic to the sphere, since on this topology all monopole loops can be contracted to a single point [15-17].

The relationship between the closed monopole loops and the discontinuity in the energy is far from being proved. The influence of those non-trivial monopole loops on the phase transition has been studied by Rebbi and coworkers [18]. Their results suggest that the properties of the phase transition seem not to be affected by the existence of such closed monopole loops. 
However, it is claimed that the two-state signal does disappear when working on a lattice constructed considering the surface of a $5 D$ cube, which has the topology of a sphere $[15,17]$. This single peak structure together with the estimation of the critical exponent $\nu \sim 0.37$ measured at $\gamma=0,-0.2,-0.5$, has induced authors in [17] to claim that the double peak signal on the torus is a finite size effect, and when working on the sphere one observes that indeed the line $\beta_{c}(\gamma)$ for $\gamma \leqslant 0$ is a line of second-order phase transitions, all of them in the same universality class characterized by a $\nu$ exponent around $1 / 3$. Assuming this second-order scenario for the pure gauge theory, fermions have been included (on the torus) and also with this topology a second-order phase transition is claimed to exist [19].

This situation is somehow disturbing, because one would expect to get the asymptotic behavior of a phase transition earlier on a lattice which is homogeneous and translationally invariant, namely the hypertorus, than on a lattice which is manifestly nonhomogeneous, as is the surface of a 5D cube, and on which the translational invariance is only recovered in the limit of infinite volume.

First-order phase transitions exhibit a wide variety of behaviors. Some of them do not show any pretransitional effect, the correlation length at the critical point, $\xi_{c}$, is small, and the thermodynamic quantities diverge as the volume for reasonable lattice sizes. However, when the correlation length is very large, pretransitional effects take place, and they can also be very significant. In literature these transitions are referred to as Weak First Order (WFO) phase transitions and are characterized by small discontinuities, such as a small latent heat.

Depending on whether or not $L$ is larger than $\xi_{c}$, a WFO phase transition presents two differentiate regions:

- Transient region $\left(L \leqslant \xi_{c}\right)$. The transition behaves like a second-order one, since $\xi_{c}$ is effectively infinite. Thermodynamic quantities diverge less than $L^{d}$ and hence the effective critical exponent $\nu$ is larger than $1 / d$.

- Asymptotic region $\left(L \gg \xi_{c}\right)$. The first-order character of the transition is revealed by the onset of a stable two-peak structure and an effective $\nu$ compatible with $1 / d$.

How large should $\xi_{c}$ be for the transition to be considered WFO seems a subjective matter. Since $\xi_{c} \neq 0$ always, all first-order transitions would be weak in some extent. The key point for a transition to be considered WFO is the existence of pretransitional effects. The pretransitional behavior makes the transition along the transient region to look like a second order one with a definite critical coupling $\beta^{\prime}$. This second-order phase transition is preempted by the true first-order one at the coupling $\beta_{c}$. The distance between the couplings $\beta^{\prime}$ and $\beta_{\mathrm{c}}$ can be taken as a measure of the weakness of the transition [20]. Typical examples of such WFO transitions are $2 D$ Potts models [20] and the deconfining transition in finite $T$ QCD [21].

From this description it is clear that the observation of no double-peak structures does not prove by itself the second-order character of a phase transition.

In view of this, we decided to make a systematic study of the phase transition line $\beta_{\mathrm{c}}(\gamma)$ on the hyper-torus (HT topology hereafter) and on the lattice with spherical 
topology following Refs. [15,17] (which will be referred to as HS topology). On the hypertorus we improve on the statistics running up to $L=24$ at some negative $\gamma$ values. On the sphere we have run lattices substantially larger than those employed by authors in [17] to check if the two-state signal reappears when larger spheres are considered. Our preliminary results, suggesting that the double peak structure indeed reappears, are published somewhere else [23]. Here, the statistics on those lattice sizes have been increased, and simulations in larger lattices are included. New observables are used to extract our conclusions.

\section{Description of the model and observables}

We shall consider the parameter space described by the extended Wilson action, which can be expressed in terms of the plaquette angle in the following way:

$$
S=-\beta \sum_{\mathrm{p}} \cos \theta_{\mathrm{p}}-\gamma \sum_{\mathrm{p}} \cos 2 \theta_{\mathrm{p}}
$$

We use for the simulations the conventional $4 \mathrm{D}$ hypercube with toroidal boundary conditions (hypertorus), and, for comparison we also consider the surface of a $5 \mathrm{D}$ cube which is topologically equivalent to a $4 \mathrm{D}$ sphere. Contrarily to the hypertorus, the surface of a $5 \mathrm{D}$ cube is not homogeneous. There are a number of sites which do not have the maximum connectivity, namely 8 neighbors. Due to this fact, uncontrolled finite size effects are expected to turn up. Their influence can be somehow alleviated by the introduction of appropriate weight factors in those inhomogeneous sites [17]. Since the topology remains unchanged and as we do not expect the order of the phase transition to be affected by the rounding, we do not use weight factors.

As a notational remark, we label with $L$ and $N$ the side length for the HT topology and for the HS topology, respectively.

Next we define the energies associated to each term in the action

$$
\begin{aligned}
E_{\mathrm{p}} & =\frac{1}{N_{\mathrm{p}}}\left\langle\sum_{\mathrm{p}} \cos \theta_{\mathrm{p}}\right\rangle, \\
E_{2 \mathrm{p}} & =\frac{1}{N_{\mathrm{p}}}\left\langle\sum_{\mathrm{p}} \cos 2 \theta_{\mathrm{p}}\right\rangle,
\end{aligned}
$$

where $N_{\mathrm{p}}$ stands for the number of plaquettes. On the hyper-torus this number is simply proportional to the volume, namely the forward plaquettes are $N_{\mathrm{p}}=6 L^{4}$. On the sphere the number of plaquettes has a less simple expression, and can be computed as a function of $N$ as $N_{\mathrm{p}}=60(N-1)^{4}+20(N-1)^{2}$. In this case, the system is not homogeneous and $N_{\mathrm{p}}$ is not proportional to the number of points on the four-dimensional surface, which is $N^{5}-(N-2)^{5}$, some points having a number of surrounding plaquettes less than the possible maximum 12 , as opposed to what happens on the torus. In order to 
allow for a comparison, we define $L_{\text {eff }}=\left(N_{\mathrm{p}} / 6\right)^{1 / 4}$, in such a way that a hypertorus of $L=L_{\text {eff }}$ has the same number of plaquettes as the corresponding hypersphere.

The specific heat and the Binder cumulant are useful quantities to monitor the properties of a phase transition. At the critical point, they are known to posses different thermodynamical limits depending on whether the transition is first order or higher order, and hence, their behavior with increasing lattice size can give some clues as to the order of the phase transition. We have studied the finite size scaling (FSS) of these two energy cumulants.

The specific heat is defined for both energies as

$$
\begin{aligned}
C_{\mathrm{v}} & =\frac{\partial}{\partial \beta} E_{\mathrm{p}}=N_{\mathrm{p}}\left(\left\langle E_{\mathrm{p}}^{2}\right\rangle-\left\langle E_{\mathrm{p}}\right\rangle^{2}\right), \\
C_{2 \mathrm{v}} & =\frac{\partial}{\partial \gamma} E_{2 \mathrm{p}}=N_{\mathrm{p}}\left(\left\langle E_{2 \mathrm{p}}^{2}\right\rangle-\left\langle E_{2 \mathrm{p}}\right\rangle^{2}\right) .
\end{aligned}
$$

As we have observed a high correlation between both energies, the results being qualitatively the same, we shall only report on the observable defined for the plaquette energy $E_{\mathrm{p}}$.

In a second-order phase transition, scaling theory predicts the specific heat maximum to diverge as $L^{\alpha / \nu}$. If the transition is first order it is expected to diverge like the volume $L^{d}$ (or strictly like the number of plaquettes $N_{\mathrm{p}}$ ) reflecting that the maximum of the energy fluctuation has the size of the volume. This is expected to hold in the asymptotic region of the transition $L \gg \xi_{c}$. In the transient region, namely $L<\xi_{c}$, the specific heat is expected to grow more slowly than the volume.

We shall use the number of plaquettes $N_{\mathrm{p}}$ to study the specific heat behavior in order to have a single parameter for both the HT and the HS topologies. This is of course equivalent to doing the discussion as a function of $L$ on the HT and $L_{\text {eff }}$ on the HS. We have in this case $C_{i} \sim\left(N_{\mathrm{p}}\right)^{\alpha / d \nu}$.

We have also studied the behavior of the fourth cumulant of the energy

$$
V_{L}=1-\frac{\left\langle E_{\mathrm{p}}^{4}\right\rangle_{L}}{\left\langle E_{\mathrm{p}}^{2}\right\rangle_{L}^{2}}
$$

When the energy distribution describing the system is gaussian $V_{L} \rightarrow 2 / 3$ in the thermodynamic limit. This is the case for a second-order phase transition. If the transition is first order, far from the critical coupling $\beta_{\mathrm{c}}, V_{L}$ also tends to $2 / 3$, reflecting the gaussianity of the energy distribution. However, at $\beta_{\mathrm{c}}$ the distribution can be described by two gaussians centered about the energy of each metastable state $E_{1}$ and $E_{2}$, and hence this quantity has the non-trivial thermodynamic limit

$$
V_{L \rightarrow \infty} \rightarrow 1-\frac{2\left(E_{1}^{4}+E_{2}^{4}\right)}{3\left(E_{1}^{2}+E_{2}^{2}\right)^{2}}<\frac{2}{3} .
$$

Another interesting quantity measurable from the density of states is the distribution of the partition function zeroes, or Lee-Yang zeroes [26]. To clarify the role of the partition function zeroes let us first stress a well-known fact: there are no phase transitions on a 
finite volume, phase transitions arise in the thermodynamical limit and are signaled by non-analyticity in the free energy,

$$
F(\beta, V)=-\frac{1}{\beta V} \log Z(\beta) .
$$

$Z(\beta)$ is a linear combination of exponentials, and hence an analytical function. This implies that the free energy can be singular only where $Z(\beta)=0$. However, if the coupling $\beta$ is real, and the volume is finite, that linear combination is a sum of positive terms and hence, the zeroes of $Z(\beta)$ are located in the complex plane of the coupling $\beta$. The onset of the phase transition in the limit of infinite volume is signaled by a clustering of the zeroes on the real axis at $\beta_{c}$.

Here follows the description of our procedure, for a general description of the method see Ref. [27].

As mentioned previously we work only with $E_{\mathrm{p}}$, and then we consider only the spectral density method [28] for this variable. Since we work at fixed $\gamma$ we consider only the $\beta$ coupling derivatives.

From the MC simulation at $\beta$ we obtain an approximation to the density of states which allows us to compute the normalized energy distribution $P_{\beta}(E)$. The energy distribution can be expressed as

$$
P_{\beta}(E)=\frac{1}{Z} W(E) e^{-\beta E}
$$

We use the standard reweighing technique to obtain from the distribution measured at $\beta$ the distribution at another coupling $\omega$, which is complex in the more general case $\omega=\eta+i \xi$. The standard reweighing formula is

$$
P_{\omega}(E)=\frac{P_{\beta}(E) e^{-(\omega-\beta) E}}{\sum_{E} P_{\beta}(E) e^{-(\omega-\beta) E}},
$$

using (11) and the normalization condition $\sum_{E} P_{\beta}(E)=1$ one obtains

$$
\frac{Z(\omega)}{Z(\beta)}=\sum_{E} P_{\beta}(E) e^{-(\omega-\beta) E} .
$$

We can factorize the contributions from the real and imaginary parts,

$$
\frac{Z(\omega)}{Z(\beta)}=\sum_{E} P_{\beta}(E)(\cos (E \xi)+i \sin (E \xi)) e^{-(\eta-\beta) E}
$$

This is the standard reweighing formula extended to the complex parameter space of couplings. As a first observation we have a pure oscillating factor due to $\operatorname{Im}(\omega) \neq 0$. Since $E$ is $O(V)$ this is a rapidly oscillating function which makes it impossible to locate zeroes with large imaginary part.

The real part of the coupling contributes to the well-known exponential damping, $e^{-(\eta-\beta) E}$, which is telling us that we can trust the extrapolation only in a small neighborhood $\beta \pm \eta$. 
Since $Z(\operatorname{Re} \omega)$ has no zeroes in a finite volume, an easy way to locate the zeroes numerically is looking at the minima of the function $|G(\omega)|^{2}$, where

$$
G(\omega)=\frac{Z(\omega)}{Z(\operatorname{Re} \omega)}=\frac{\sum_{E} P_{\beta}(E)(\cos (E \xi)+i \sin (E \xi)) e^{-(\eta-\beta) E}}{\sum_{E} P_{\beta}(E) e^{-(\eta-\beta) E}} .
$$

The function to minimize is

$$
|G(\omega)|^{2}=\frac{\left(\sum_{E} P_{\beta}(E) e^{-(\eta-\beta) E} \cos (E \xi)\right)^{2}+\left(\sum_{E} P_{\beta}(E) e^{-(\eta-\beta) E} \sin (E \xi)\right)^{2}}{\left(\sum_{E} P_{\beta}(E) e^{-(\eta-\beta) E}\right)^{2}}
$$

One interesting property of the partition function zeroes concerns the estimation of critical exponents. Denoting by $\omega_{0}$ the coupling where the first zero is located, the distance to the real axis scales with the $\nu$ exponent,

$$
\operatorname{Im}\left(\omega_{0}\right) \sim L^{-1 / \nu}
$$

We shall use this property to compute the effective $\nu$ exponent.

From $P_{E}(\beta)_{L}$, we can measure the free energy gap, $\Delta F(L)$, which is the difference between the minima and the local maximum of the free energy [24]. We use the spectral density method to get, from the measured histograms, a new histogram where both peaks have equal height. We take the logarithm of those histograms and measure the energy gap. A growing $\Delta F(L)$ in the asymptotic region of the transition implies a first-order phase transition. An increase proportional to $L^{d-1}$ is expected [24]. For the transition to be second order, $\Delta F(L)$ must stay constant with increasing lattice sizes.

\section{Numerical simulation}

Most of the work has been done by simulating the subgroup $Z(1024) \subset U(1)$, since the phase transition associated to the discrete group lies safely far away. The overrelax algorithm can be applied only in the simulations with $\gamma=0$ so that the gain in statistical quality due to the overrelax effect is limited to the Wilson action, where we have simulated both the full group $U(1)$ and the discrete one for the sake of comparison. For $\gamma \neq 0$ we have simulated the discrete group as it considerably speeded up the simulation.

For every lattice size we consider, we perform trial runs to locate the coupling $\beta^{*}(L)$ where the specific heat shows a peak. We use the standard reweighing techniques to extrapolate in a neighborhood of the simulated coupling. Once the peak is located within an error in the fourth digit of the coupling, we perform an intensive simulation there to get $P_{E}\left(\beta^{*}\right)_{L}$. The statistics performed at these pseudo-critical couplings are reported in Tables 1 and 2 for the HS and the HT topology respectively.

Typically we measure the energies every $10 \mathrm{MC}$ sweeps in order to construct the energy histogram (11). From this energy distribution we obtain the cumulants of the 
Table 1

Statistics of the data obtained for the HS topology

\begin{tabular}{|c|c|c|c|c|c|c|}
\hline$N$ & $L_{\mathrm{eff}}$ & $\beta_{\text {sim }}$ & $\tau$ & $N_{\tau}$ & $\beta^{*}(L)$ & $\operatorname{Im}\left(\omega_{0}\right)$ \\
\hline \multicolumn{7}{|c|}{$\gamma=+0.2$} \\
\hline 4 & 5.383 & 0.8910 & 70 & 29142 & $0.8906(2)$ & $0.0169(1)$ \\
\hline 5 & 7.150 & 0.8855 & 210 & 8000 & $0.8855(2)$ & $0.00604(6)$ \\
\hline 6 & 8.921 & 0.8834 & 520 & 3800 & $0.88330(5)$ & $0.00244(6)$ \\
\hline 7 & 10.694 & 0.8818 & 790 & 2300 & $0.88182(3)$ & $0.00090(3)$ \\
\hline 8 & 12.469 & 0.88095 & 930 & 1930 & $0.88097(3)$ & $0.00040(2)$ \\
\hline \multicolumn{7}{|c|}{$\gamma=0$} \\
\hline 6 & 8.921 & 1.0128 & 240 & 8300 & $1.01340(5)$ & $0.00616(8)$ \\
\hline 8 & 12.469 & 1.0125 & 400 & 2300 & $1.0127(2)$ & $0.00196(4)$ \\
\hline 10 & 16.021 & 1.0120 & 780 & 1200 & $1.01212(3)$ & $0.00075(3)$ \\
\hline 12 & 19.574 & 1.0119 & 850 & 1150 & $1.01194(2)$ & $0.00031(2)$ \\
\hline 14 & 23.123 & 1.0117 & 920 & 1900 & $1.01168(2)$ & $0.00013(1)$ \\
\hline \multicolumn{7}{|c|}{$\gamma=-0.2$} \\
\hline 6 & 8.921 & 1.1587 & 160 & 2000 & $1.1587(4)$ & $0.0107(3)$ \\
\hline 7 & 10.694 & 1.1600 & 320 & 2800 & $1.1597(1)$ & $0.00550(7)$ \\
\hline 8 & 12.469 & 1.1597 & 510 & 1000 & $1.1603(2)$ & $0.00321(4)$ \\
\hline 10 & 16.021 & 1.1602 & 680 & 1500 & $1.1604(2)$ & $0.00171(2)$ \\
\hline 12 & 19.574 & 1.1604 & 820 & 1200 & $1.1602(1)$ & $0.00083(1)$ \\
\hline 14 & 23.123 & 1.1605 & 900 & 1100 & $1.16048(5)$ & $0.00044(1)$ \\
\hline 16 & 26.684 & 1.1604 & 1150 & 1200 & $1.16038(2)$ & $0.00023(1)$ \\
\hline
\end{tabular}

energy we are interested in, and the critical exponents using finite size scaling techniques. We remark that one of the main sources of systematic error when measuring critical exponents is the indetermination in the coupling $\beta^{*}(L)$ where to measure.

The simulations have been done in the RTNN machine consisting of 32 Pentium Pro $200 \mathrm{MHz}$ processors. The total CPU time used is the equivalent of 6 Pentium Pro years.

We have updated using a standard Metropolis algorithm with 2 hits. The acceptance has always been between $65 \%$ and $75 \%$.

In order to consider the statistical quality of the simulation, following Ref. [29] we define the unnormalized autocorrelation function for the energy

$$
C(t)=\frac{1}{N-t} \sum_{i=1}^{N-t} E_{i} E_{i+t}-\langle E\rangle^{2},
$$

as well as the normalized one

$$
\rho(t)=\frac{C(t)}{C(0)} .
$$


Table 2

Statistics of the data obtained in the HT topology

\begin{tabular}{|c|c|c|c|c|c|}
\hline$L$ & $\beta_{\mathrm{sim}}$ & $\tau$ & $N_{T}$ & $\beta^{*}(L)$ & $\operatorname{Im}\left(\omega_{0}\right)$ \\
\hline \multicolumn{6}{|c|}{$\gamma=-0.1$} \\
\hline 6 & 1.0720 & 350 & 1900 & $1.0716(2)$ & $0.0097(1)$ \\
\hline 8 & 1.0784 & 640 & 1400 & $1.0786(2)$ & $1.1539(2)$ \\
\hline 12 & 1.0820 & 820 & 750 & $1.0818(1)$ & $0.00114(2)$ \\
\hline 16 & 1.08278 & 930 & 900 & $1.0827(1)$ & $0.00040(2)$ \\
\hline 20 & 1.0833 & 1150 & 1100 & $1.0833(1)$ & $0.00020(1)$ \\
\hline \multicolumn{6}{|c|}{$\gamma=-0.2$} \\
\hline 6 & 1.1460 & 380 & 2000 & $1.1452(2)$ & $0.0115(1)$ \\
\hline 8 & 1.1535 & 620 & 1900 & $1.1539(2)$ & $0.00506(6)$ \\
\hline 12 & 1.1582 & 840 & 1200 & $1.1582(2)$ & $0.00152(3)$ \\
\hline 16 & 1.15935 & 920 & 900 & $1.1593(1)$ & $0.00060(2)$ \\
\hline 20 & 1.1599 & 1150 & 900 & $1.1599(1)$ & $0.00028(1)$ \\
\hline \multicolumn{6}{|c|}{$\gamma=-0.3$} \\
\hline 6 & 1.2255 & 340 & 2000 & $1.2237(4)$ & $0.0138(1)$ \\
\hline 8 & 1.2344 & 560 & 1800 & $1.2340(1)$ & $0.00623(4)$ \\
\hline 12 & 1.2395 & 770 & 1000 & $1.2395(2)$ & $0.00190(3)$ \\
\hline 16 & 1.2410 & 900 & 900 & $1.2410(1)$ & $0.00084(2)$ \\
\hline 20 & 1.2416 & 1100 & 1200 & $1.24156(5)$ & $0.00041(2)$ \\
\hline 24 & 1.2417 & 1200 & 1100 & $1.24162(5)$ & $0.00022(1)$ \\
\hline \multicolumn{6}{|c|}{$\gamma=-0.4$} \\
\hline 6 & 1.3090 & 400 & 1800 & $1.3082(4)$ & $0.0152(1)$ \\
\hline 8 & 1.3192 & 600 & 1600 & $1.3194(3)$ & $0.00704(5)$ \\
\hline 12 & 1.3258 & 710 & 1400 & $1.3259(1)$ & $0.00238(3)$ \\
\hline 16 & 1.32775 & 840 & 900 & $1.3278(1)$ & $0.00108(2)$ \\
\hline 20 & 1.3285 & 930 & 1600 & $1.3284(1)$ & $0.00054(2)$ \\
\hline 24 & 1.3286 & 1150 & 1500 & $1.3286(1)$ & $0.00029(1)$ \\
\hline
\end{tabular}

The integrated autocorrelation time for the energy, $\tau^{\text {int }}$, can be measured using the window method,

$$
\tau^{\mathrm{int}}(t)=\frac{1}{2}+\sum_{t^{\prime}=1}^{t} \rho\left(t^{\prime}\right),
$$

for large enough $t$, which is in practice selected self-consistently. We use $t$ in the range $5 \tau^{\mathrm{int}}, 10 \tau^{\mathrm{int}}$, and we check that the obtained $\tau^{\mathrm{int}}$ remains stable as the window in $t$ is increased.

We have always started from hot and cold configurations in order to make sure that the system does not remain in a long living metastable state, which could be 
interpreted as a Dirac sheet. The results coming from both types of starts have always been indistinguishable.

\section{The phase transition line $\beta_{c}(\gamma)$}

We have studied the deconfinement-confinement phase transition line at several values of $\gamma$ (Fig. 1).

In the region of negative $\gamma$, apart from the deconfinement transition line, there is another transition line provoked by the competing interaction between the couplings $(\beta, \gamma)$. In the limit $\gamma=-\infty$ the model is not dynamical since for all finite $\beta, \cos \theta_{p}=0$. So we expect this line to end at the corner $(\gamma=-\infty, \beta=+\infty)$. We have not performed a deep study of this transition line, however the simulations at $L=8$ revealed doublepeak structures pointing to a first-order character. The limit $\gamma=+\infty$ is equivalent to a $\mathbb{Z}(2)$ theory and the critical point can be calculated exactly by self-duality.

We have carefully studied the region between lines $A$ and $B$ finding no signatures of the existence of additional lines.

We have focused on the transition line $A$, at several values of $\gamma$, on the hyper-torus and on the hyper-sphere.

The structure of this section is the following. First, we describe the results at $\gamma=+0.2$ on the spherical topology. Our purpose is to observe how does the HS topology behave in a non-controversial region, and comparing with the known results on the hypertorus.

Second, we study the point $\gamma=0$ (Wilson action) on the spherical lattice. This coupling has been recently claimed to be the starting point of a critical line (infinite correlation length at the critical point) which extends in the range $\gamma \leqslant 0$, with an associated critical exponent $\nu \sim 0.37$ [17]. Since this assertion relies on the absence of two-state signals on spherical lattices up to $N=10$, we shall check whether or not double peak structures set in when larger spheres are considered.

Third, we go to the region of negative $\gamma$. We are aware of no previous systematic study on the hypertorus in this region, so we run simulations up to $L=24$ at $\gamma=-0.1$, $-0.2,-0.3,-0.4$. Motivated by the results on the hyper-torus we have just run a single $\gamma$ negative value on the hyper-sphere. For the sake of comparison with Ref. [17] we choose this value to be $\gamma=-0.2$.

Finally, we discuss the finite size scaling behavior exhibited by both topologies.

\subsection{Results at $\gamma=+0.2$ on the spherical topology}

As we have pointed out, there is a general agreement on considering the phase transition first order in the region of positive $\gamma$.

The lattice sizes used range from $N=4$ to $N=8$, which correspond to $L_{\text {eff }} \sim 5$ and $L_{\text {eff }} \sim 12$ respectively see (Table 1 ).

In Fig. 2, the MC evolution for $N=6,7,8$ is shown. We remark that no multicanonical update is needed to obtain a very high rate of flip-flops up to $N=8$. However, on the 


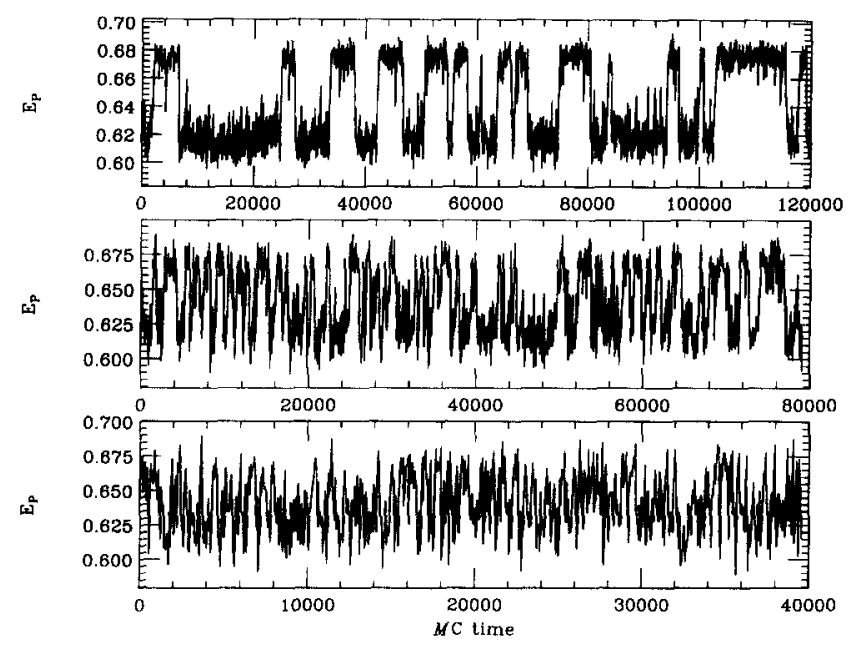

Fig. 2. MC evolution of $E_{\mathrm{p}}$ at $\gamma=+0.2$ on the HS topology for $N=6$ (lower window), $N=7$ (middle) and $N=8$ (top).

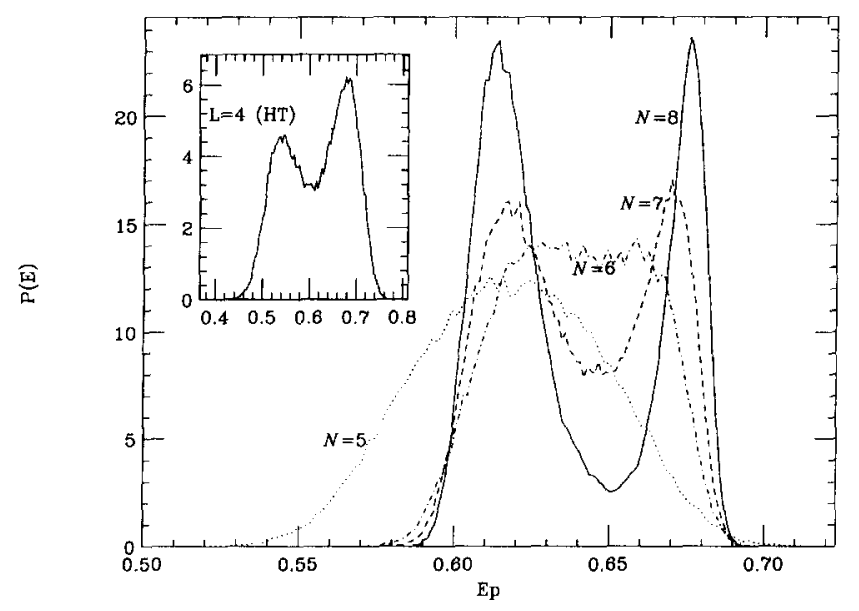

Fig. 3. $E_{\mathrm{p}}$ distribution at $\gamma=+0.2$ on the HS topology. The small window is the distribution we obtained on the HT topology in $L=4$ at $\beta=0.8595$.

torus, for $L>6$ the probability of tunneling between both metastable states is so tiny that a reasonable rate of flip-flops is not accessible to ordinary algorithms. Probably the inhomogeneity of the sphere is increasing the number of configurations with energies which correspond neither to the confined nor to the deconfined phase, but in between. These configurations make the free energy gap to decrease and hence the tunneling is easier on the HS topology. In short, the sites without maximum connectivity act as catalysts of the tunneling.

In Fig. 3 the energy distributions are plotted. The distribution at $N=6$ is distinctly non-gaussian, but a blatant two-peak structure is observed only from $N=7\left(L_{\text {eff }} \sim 11\right)$ on. So, when comparing with the result at $L=4$ on the torus (see the small window in 


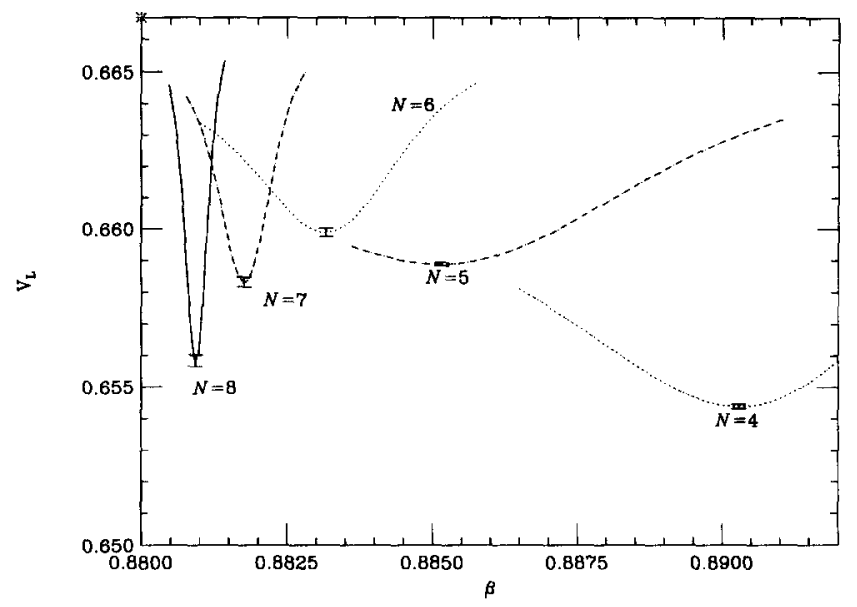

Fig. 4. Binder cumulant at $\gamma=+0.2$ on the HS topology in $N=4,5,6,7,8$. The cross in the upper corner signals the second-order value $2 / 3$.

Fig. 3) a first observation is that the onset of a two-state signal is particularly spoiled by the HS topology, at least in $4 D$ pure compact $U(1)$ gauge theory.

Concerning the latent heat, we remark its stability already at $N=7$, or, if anything, its increase from $N=7$ to $N=8$. One would even say that the same happens between the positions of the would-be two states in $N=6$ and the position observed in $N=7$. The behavior of the Binder cumulant reflects this fact (see Fig. 4). We observe a rapid growth of $V_{L}^{\min }$ for small lattices sizes, apparently towards $2 / 3$ (second-order value). This growth stops when the splitting of the two peaks is observed. The splitting of the two peaks is reflected by smaller values in $V_{L}^{\min }$. We remark that the errors quoted for $V_{L}^{\min }$ are calculated taking into account the indetermination in the value of the minimum, but not the displacement in the position of the coupling where the minimum appears.

In our opinion, mainly two reasons can account for this behavior. The first one comes from general grounds: at the very asymptotic region of a first-order phase transition, the energy jump gets sharper and sharper, and a slightly increase of the latent heat could be expected. The second one would be the increasing restoration of homogeneity in the hypersphere when increasing the lattice size. The latent heat observed in small $N$ might be affected by the inhomogeneity of the hyper-sphere.

At this point we cannot give a single reason for this to happen. We postpone a stronger conclusion to the section devoted to the Finite Size Scaling discussion.

The value of the latent heat when obtained with a cubic spline fit to the peaks in $N=8$ is $C_{\text {lat }}=0.064(2)$. Results obtained with mixed hot-cold starts in $N=9$ seems to give an energy jump around 0.067 . However, the reliability of this method is very limited and we do not dare to extract strong conclusions from it. Taking into account that the cubic spline at $N=7$ gives $0.053(2)$, a possible scenario would be a slowly increasing latent heat towards its asymptotic value $C_{\mathrm{lat}}(\infty)$.

The peak of the specific heat for the different lattice sizes is displayed in Fig. 5. The 


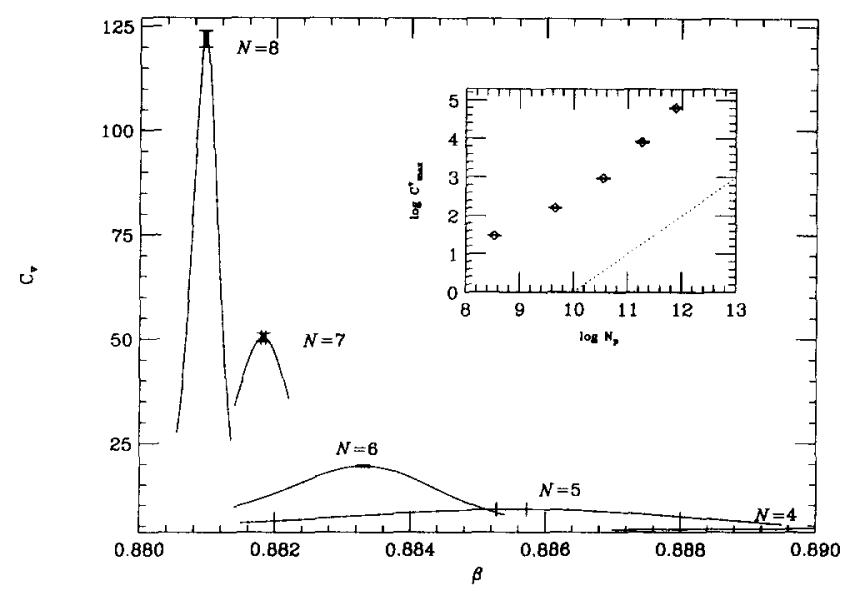

Fig. 5. Specific heat maximum, and Ferrenberg-Swendsen extrapolation (solid line) at $\gamma=+0.2$ on the HS topology. The small window represents $C_{\mathrm{v}}^{\max }\left(N_{\mathrm{p}}\right)$ The dotted line corresponds to the behavior expected in a first-order phase transition.

continuous line represents the $F S$ extrapolation. The plot of the peak value, $C_{v}^{\max }\left(N_{\mathrm{p}}\right)$ as a function of the plaquette number reveals a linear relationship, and hence a first-order character.

\subsection{Results at $\gamma=0$ on the spherical topology}

Let us first situate the status of the studies using the toroidal topology with the Wilson action. As we have pointed out in the introduction, the transition was believed to be continuous till the simulation of a $L=6$ lattice revealed the first signs of the existence of two metastable states [4]. Numerical simulations up to $L=10$ showed a rapid decrease of the latent heat when increasing the lattice size, suggesting that the two metastable states might superimpose in the limit $L \rightarrow \infty$ [5]. However, in [30], Azcoiti et al. suggest that the latent heat starts to stabilize at $L \sim 12,14$.

When one tries to simulate large lattices the tunneling becomes scarce and the use of multicanonical simulations for those lattice sizes seems to be in order. However, in spite of its usefulness in spin models, we are aware of no multicanonical simulation showing a substantial flip-flop rate improvement for this model. An alternative procedure to enhance the tunneling probability has been proposed in [22]. Within this approach the use of a monopole term in the action as a dynamical variable has been revealed as a useful method to increase the flip-flop rate.

Lattice sizes up to $L=16$ have also been studied using multihistogramming techniques [6] and RG approaches [9,10]. The results support the idea of a quasi-stable latent heat for $L>12$.

Topological considerations stressed already in the introduction led some authors to use lattices homotopic to the sphere. In the case of the Wilson action $(\gamma=0)$ the two-state signal is absent up to $N=10$ [17]. Following this, we have simulated on the spherical 


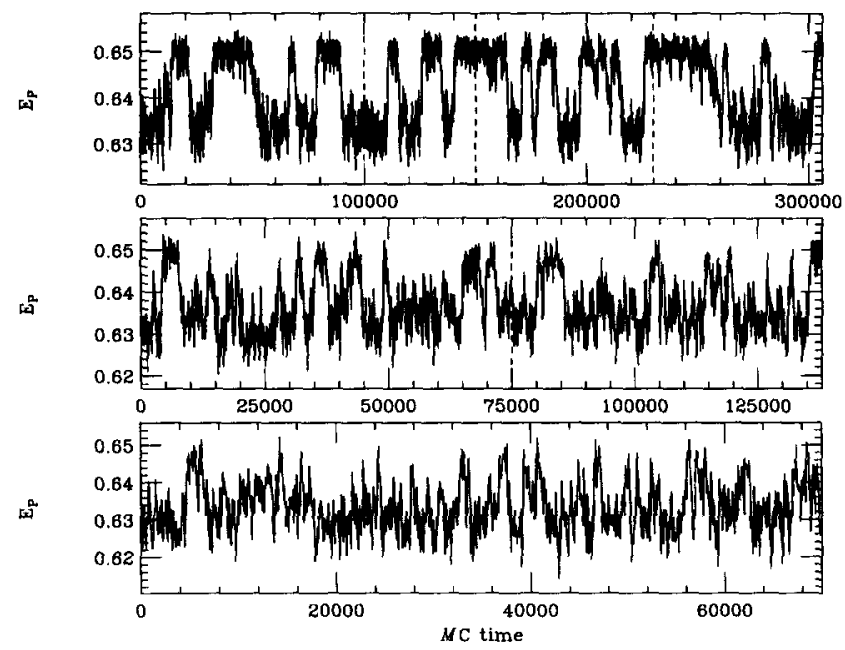

Fig. 6. MC evolution of $E_{\mathrm{p}}$ at $\gamma=0$ on the HS topology for $N=10$ (lower window), $N=12$ (middle) and $N=14$ (top). The vertical dashed lines separate different runs.

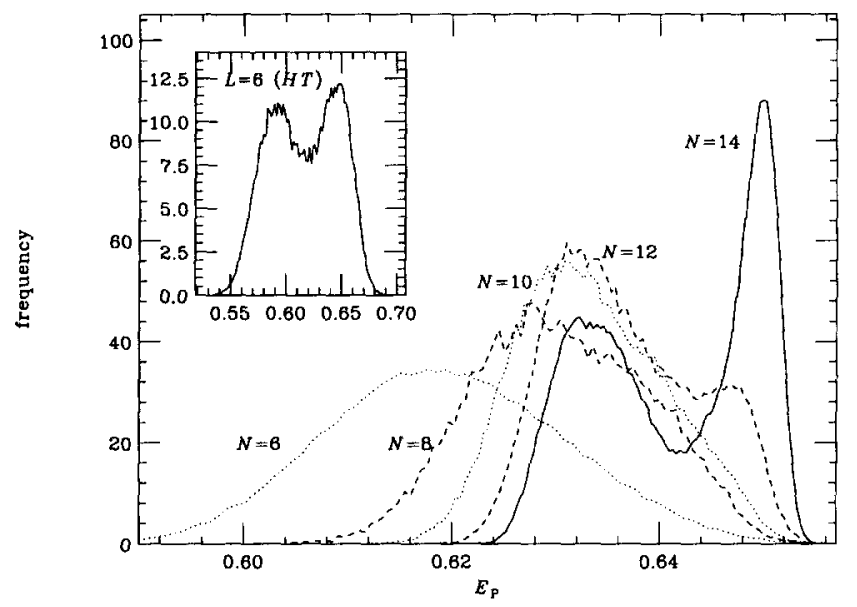

Fig. 7. $E_{\mathrm{p}}$ distribution at $\gamma=0$ on the HS topology. The small window corresponds to our simulation in the HT topology in $\mathrm{L}=6$ at $\beta=1.0020$.

topology in lattices ranging from $N=6$ to $N=14$, finding that the two-state signal sets in from $N=12 \mathrm{on}$. The preliminary results up to $N=12$ are already published [23]. We include here the results with higher statistics in $N=12$ and the results in $N=14$.

The MC evolution for $N=10,12,14$ is plotted in Fig. 6 . For the $N=14$ lattice we have four independent runs, signaled by the dashed lines in the figure, all of them giving the same predictions.

In Fig. 7 the distribution of $E_{\mathrm{p}}$ is plotted in lattices ranging from $N=6$ to $N=14$. We observe that a two-peak structure is revealed first time by the histogram in $N=12$, which has a $L_{\text {eff }} \sim 19$. On the toroidal topology the equivalent signal is observed already at $L=6$ (see small window in Fig. 7). 


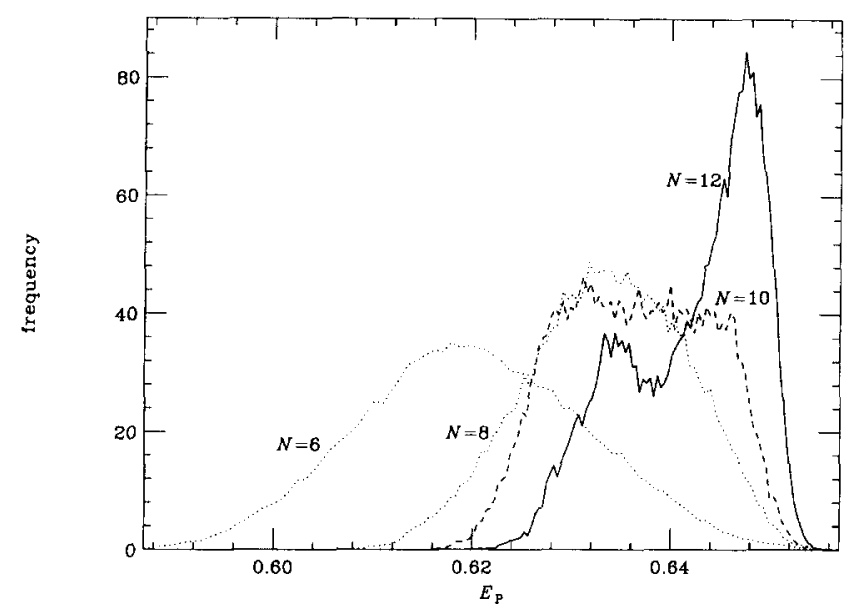

Fig. 8. $E_{\mathrm{p}}$ distribution at $\gamma=0$ on the HS topology simulating the full group $\mathrm{U}(1)$.

Having in mind the results of the previous section at $\gamma=+0.2$, it seems that the minimum $L_{\text {eff }}$ required to observe a two-peak structure in the spherical topology is around three times the minimum lattice size $L$ needed in the torus to observe two peaks.

The lattice sizes used in [17] at $\gamma=0$ ranged from $N=4$ to $N=10$, so it is not surprising that they did not see any two-peak structure. Our results are in that sense compatible with theirs, though ours show a faster divergence for $C_{\mathrm{v}}^{\max }(N)$ with increasing $N$ than the one observed in [17] in the lattice sizes we share $(N=6,8,10)$. However, we are particularly confident on this point because our simulation has been performed closer to the peak of the specific heat $\beta^{*}(L)$, and so we expect the Ferrenberg-Swendsen extrapolation to be more precise.

For the sake of comparison with the full group $U(1)$, simulations with the Wilson action have been performed at $\gamma=0$. At this coupling an implementation of overrelax is possible, and the global decorrelating effect should manifest itself in a better statistical quality.

In Fig. 8 we show the histograms of $E_{\mathrm{p}}$ from $N=6$ to $N=12$ simulating the full group. The results are fully compatible with those obtained from simulations with $Z(1024)$.

This test being performed, we go back to the description of the results obtained for the discrete group.

In Fig. 9 we plot $C_{\mathrm{v}}(N)$ for different $N$ 's, together with the Ferrenberg-Swendsen extrapolation. The small window is a $\log -\log$ plot of $C_{v}^{\max }(N)$ as a function of $N_{\mathrm{p}}$. A linear behavior is observed from $N=10 \mathrm{on}$, which is even faster than linear when $N=14$ is taken into account. This fact by itself implies the first-order character of the transition since it means that the maximum in the energy fluctuation has the size of the volume. As a further check we have measured the $\nu$ exponent from the scaling of the Fisher zeroes (see Table 1).

In Fig. 10 we plot $|G(\omega)|^{2}$ for several lattice sizes on the spherical topology at $\gamma=0$. 


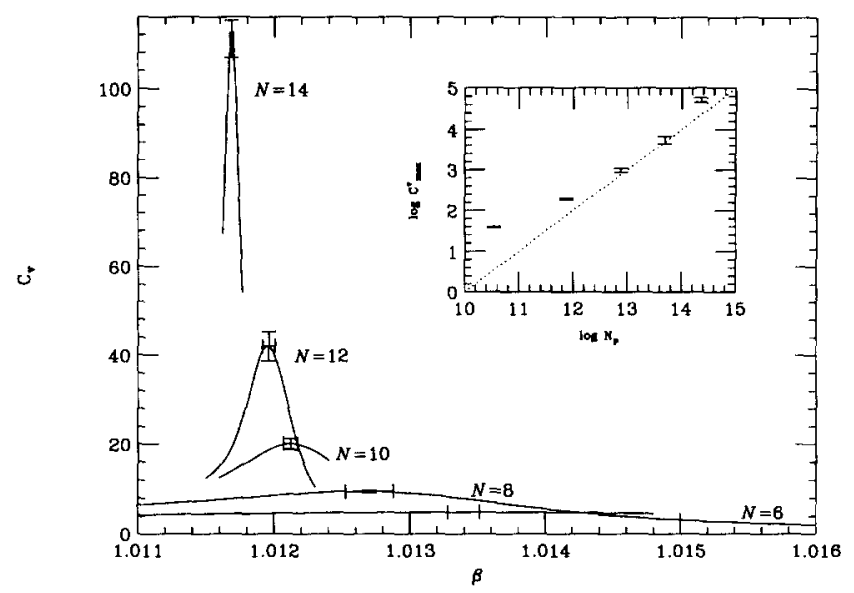

Fig. 9. Specific heat maximum, and Ferrenberg-Swendsen extrapolation (solid line) at $\gamma=0$ on the HS topology. The small window represents $C_{\mathrm{v}}^{\max }\left(N_{\mathrm{p}}\right)$ The dotted line corresponds to the behavior expected in a first-order phase transition.

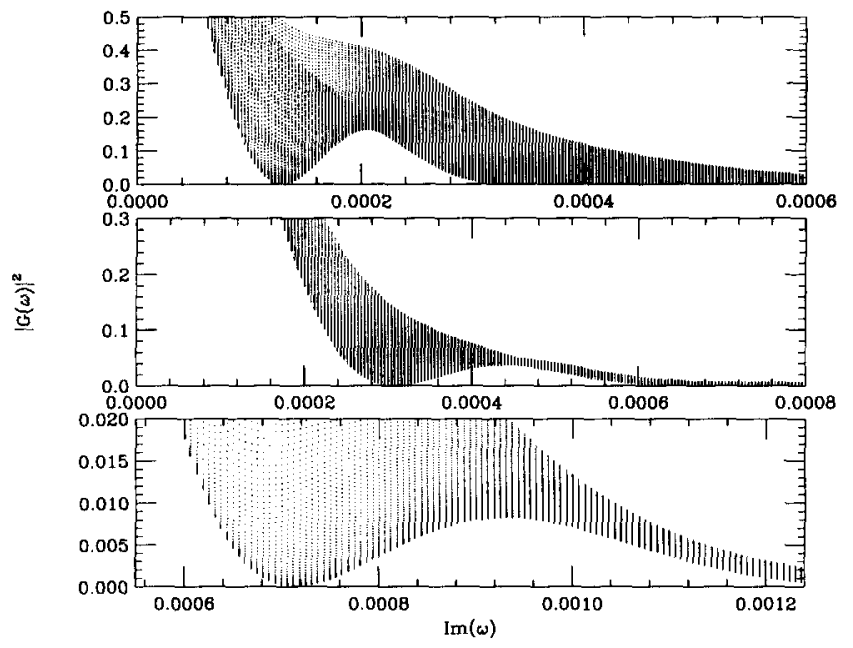

Fig. 10. Plot of (16) for $N=10$ (lower window), $N=12$ (middle) and $N=14$ (top) at $\gamma=0$ in the HS topology.

The different curves stand for the different $\omega$ we extrapolate. For small lattice sizes $\operatorname{Im}\left(\omega_{0}\right)$ is larger, and the damping is more severe than for the larger lattices since in the later the imaginary part contributes with a faster oscillating function. Actually, we observe that for $N=14$ even a second minimum could be measured before the signal is damped, while for $N=10$ one can measure accurately only the first one.

The results for the effective $\nu$ are plotted in Fig. 11 lower window. As could be expected from the behavior of the specific heat, for small lattices the effective exponent is somewhat larger than 0.25 . It gets compatible with the first-order value from $N=10$ on. In the largest lattices the distance between the two peaks slightly increases, and 


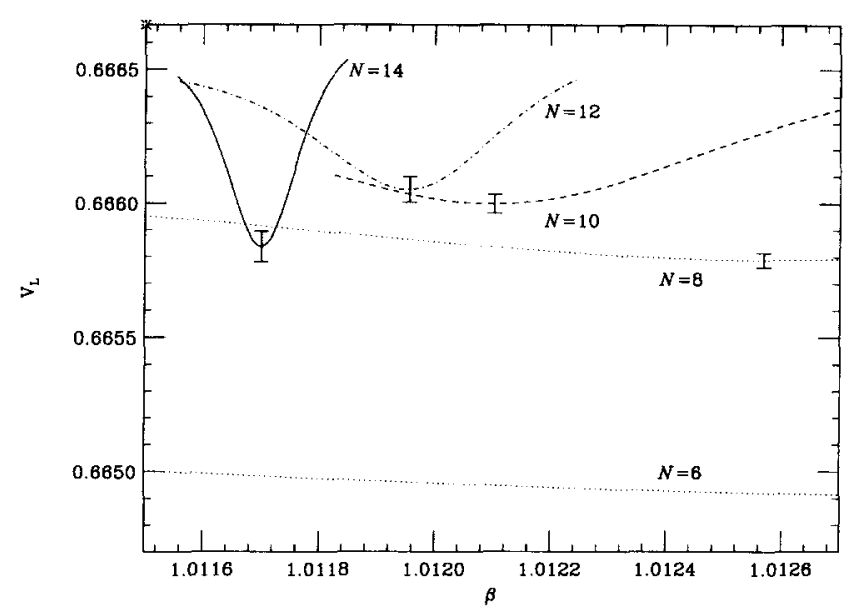

Fig. 11. Effective exponent $\nu$ at $\gamma=0$ (lower window), and at $\gamma=-0.2$ (upper window) on the spherical topology. In the smaller lattices an $\nu_{\text {eff }} \sim 1 / 3$ is observed which becomes $1 / d$ when large enough lattices are considered.

we measure a $\nu_{\text {eff }}$ slightly smaller than the first-order value. As a $\nu<0.25$ is strictly impossible we expect this to be a transient effect due to finite size effects associated to the observed splitting up of the two peaks.

Concerning the latent heat, we observe a behavior completely analogous to the one observed at $\gamma=+0.2$. We do observe a two peak structure quite stable when comparing $N=12$ and $N=14$, but it slightly increases in $N=14$. The plot of the Binder cumulant reflects again this fact (see Fig. 12). The fast growth of $V_{L}^{\min }$ towards $2 / 3$ is preempted by the onset of double peaked histograms from $N=12$ on, and it even decreases in $N=14$.

The cubic spline fit in $N=14$ at the peaks gives for the latent heat $C_{\text {lat }} \approx 0.018(2)$. which is compatible with the results suggested by extrapolating to infinite volume the values obtained on the torus up to $L=14$. Our results are hence supporting the conjecture stressed in [30] about a quasi-stabilization of the latent heat on the torus from $L=12$ on.

\subsection{Results for $\gamma<0$}

\subsubsection{Toroidal topology}

We have performed a systematic study of the transition at several negative values of $\gamma$ on the toroidal topology.

We find that the two-state signal persists for all $\gamma$ values we consider. The energy histograms reveal an increasing weakness of the transition when going to more negative $\gamma$ values (see Fig. 13). A double peak structure is clearly visible at $\gamma=-0.1$ in $L=8$, while at $\gamma=-0.4$ one has to go to $L=12$ to observe an equivalent signal.

In what concerns the behavior of the free energy gap $\Delta F(L)$, it grows for all investigated lattice sizes at all negative $\gamma$ values (see Fig. 14 lower window). The value of 

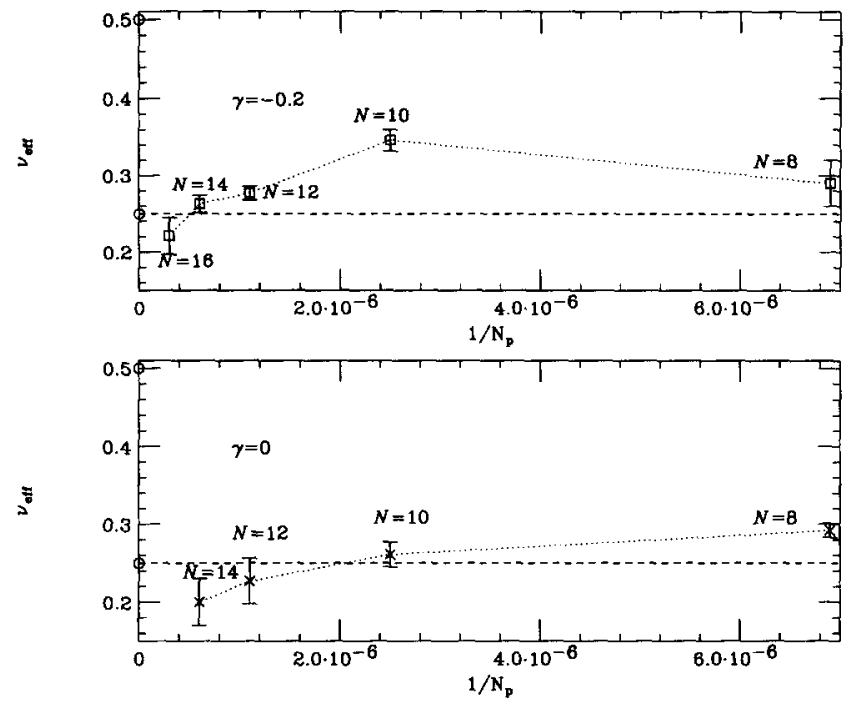

Fig. 12. Binder cumulant at $\gamma=0$ (Wilson action) on the HS topology in $N=7,8,10,12,14$. The cross in the upper corner signals the second-order value $2 / 3$.
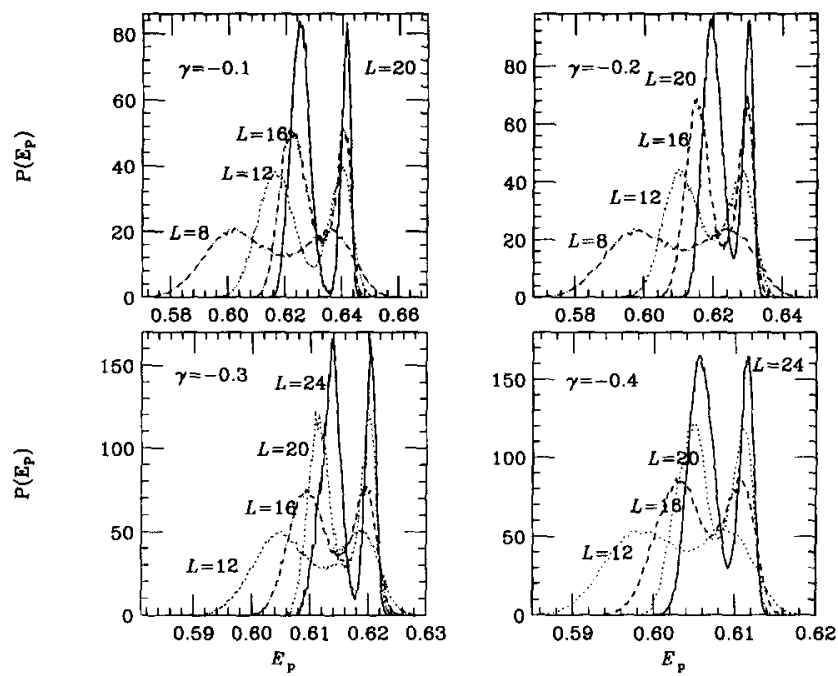

Fig. 13. $E_{\mathrm{p}}$ distributions at the different negative $\gamma$ on the HT topology. We have run up to $L=20$ at $\gamma=-0.1,-0.2$ and up to $L=24$ at $\gamma=-0.3,-0.4$.

$L$ at which $\Delta F(L)$ starts growing is certainly larger as the value of $\gamma$ is more negative. This is another test of the increasing weakness of the transition as $\gamma$ gets more negative.

The statistics performed on the torus are reported in Table 2 . We also quote for the different negative $\gamma$, the value of $\beta^{*}(L)$ and the position of partition function zero closest to the real axis. We have computed from the imaginary part of the zeroes the effective $\nu$ exponent between consecutive lattice sizes following (17).

In Fig. 15 we plot for the different $\gamma$ values the $\nu_{\text {eff }}$ we measure. In all cases a 


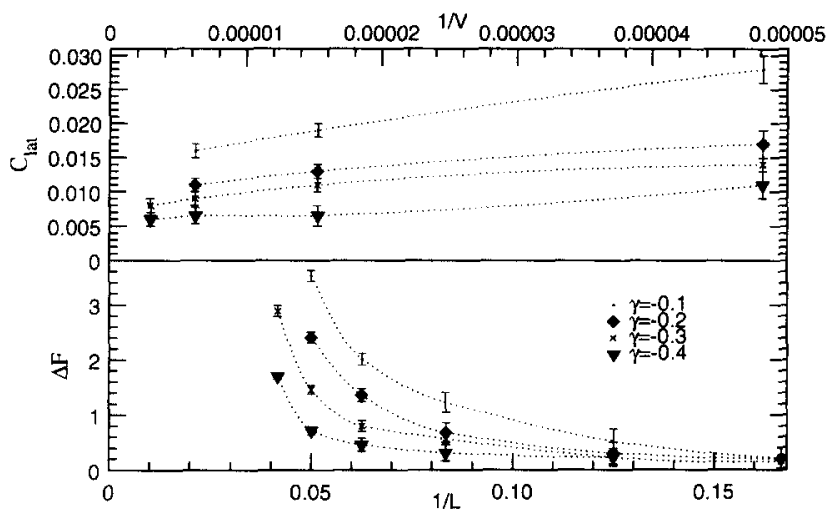

Fig. 14. Free energy gap $\Delta F(L)$ (lower window) and latent heat (upper window) for the different negative $\gamma$ in the HT topology.
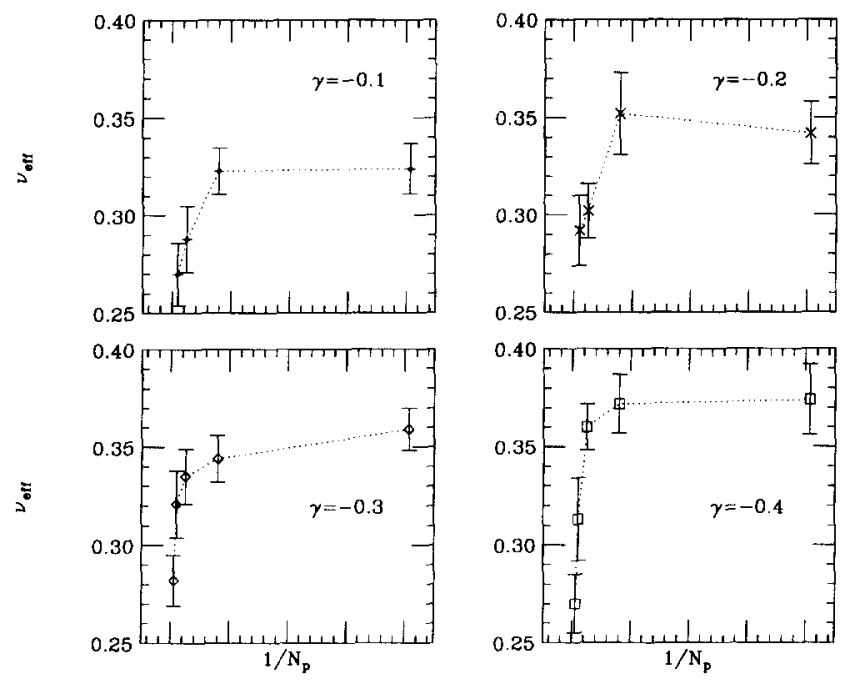

Fig. 15. Effective $\nu$ exponent for the different negative $\gamma$ in the HT topology.

$\nu_{\text {eff }} \sim 1 / 3$ is observed for small lattice sizes, which gets closer to 0.25 when the lattice is large enough. From this figure the trend of $\nu_{\text {eff }}$ seems rather clear towards the first-order value.

From the energy distributions we measure the latent heat through a cubic spline at the peaks. Taking into account the value $\nu_{\text {eff }}=0.25$ we measure, we plotted it as a function of the inverse of the volume $L^{-4}$, which is also the expected behavior of the latent heat when the transition is first order. The latent heat can be extrapolated to a value which is safely far from zero (see Fig. 14 upper window).

In Fig. 16 we plot $C_{v}^{\max }(L)$ as a function of $N_{\mathrm{p}}$. As could be expected from the behavior of the effective exponent $\nu$, the maximum of the specific heat for small lattices diverges slower than the volume. For the smaller lattices the effective exponent is 


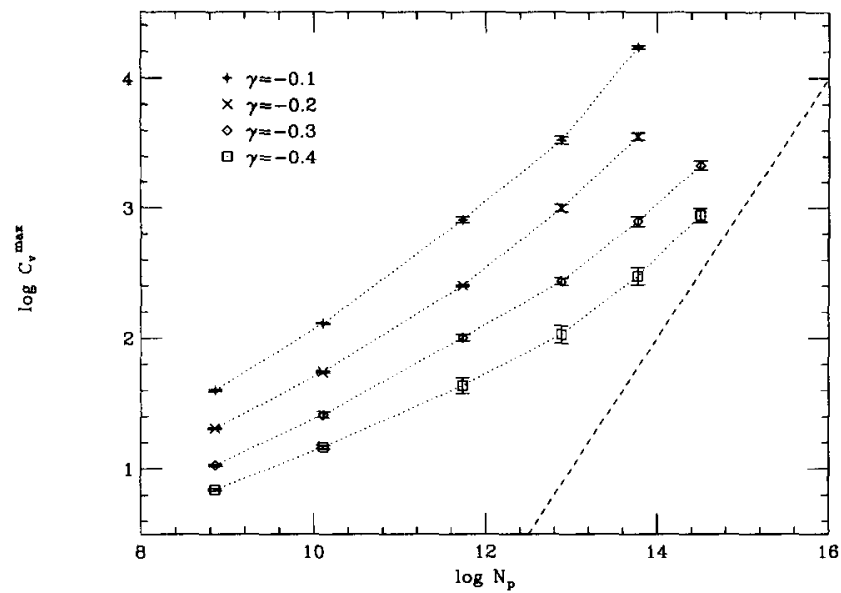

Fig. 16. $C_{v}^{\max }(L)$ as a function of $N_{\mathrm{p}}$ for the different negative $\gamma$ values on the HT topology. We have used $\log -\log$ scale for the clarity of the graphic sake. The dotted line corresponds to the first-order behavior.

$\alpha / \nu \sim 1.4$. This value increases monotonically with the lattice size. In the largest ones we observe $\alpha / \nu \sim 3.5$.

\subsubsection{Spherical topology}

On the toroidal topology we have found that the minimum lattice size required to observe a two-state signal is obtained through an appropriate combination $\left(\gamma, L_{\min }\right)$, with increasing $L_{\min }$ for increasingly negative $\gamma$, the behavior being qualitatively similar for all the $\gamma$ values we have investigated. In view of this, we have studied a single $\gamma<0$ value on the spherical lattice to check if the two-state signal is absent on this topology. For the sake of comparison with Refs. $[15,17]$ we choose this value to be $\gamma=-0.2$.

We have run simulations on spheres ranging from $N=6\left(L_{\text {eff }} \sim 8\right)$ to $N=16$ ( $L_{\text {eff }} \sim 26$ ). The MC evolution is plotted in Fig. 17.

The distribution in $N=14$ is distinctly non-gaussian and the splitting of the peaks occurs in $N=16$ (see Fig. 18). We remark that the simulations in $N=16$ are extremely expensive in CPU. In order to alleviate thermalization we have parallelized the code using shared memory in two PPro processors. In this lattice we have run two independent simulations starting from different configurations the results being fully compatible.

The behavior of the maximum of the specific heat is shown in Fig. 19. We observe the same trend than in the previous values of $\gamma$ in the sphere, i.e. an increasingly fast divergence of the specific heat with the lattice size, showing an effective $\alpha / \nu \sim 4$ already when the histogram width becomes constant with increasing lattice size.

At this value of $\gamma$ we have a worse estimation for the latent heat to be, as the two peaks have not split enough to allow for an accurate measurement.

Again the behavior of the Binder cumulant is very significant (see Fig. 20). The value $V_{L}^{\min }$ shows a very fast trend towards $2 / 3$ in the small lattices. When increasing the lattice size the rate gets slower, and finally the value in $N=16$ is compatible with 


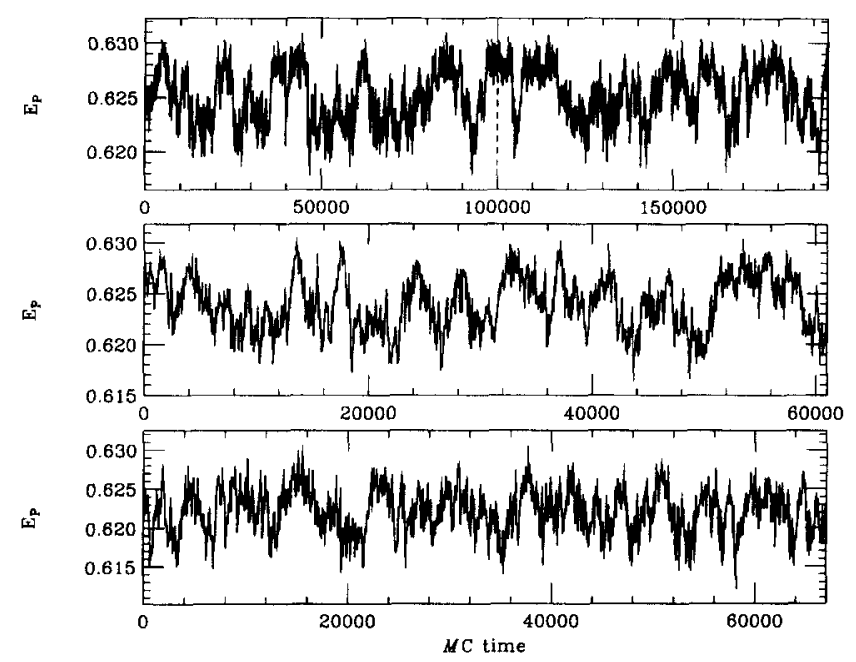

Fig. 17. MC evolution of $E_{\mathrm{p}}$ in $N=12$ (lower window), $N=14$ (middle window) and $N=16$ (upper window) at $\gamma=-0.2$ on the HS topology. The two different runs in $N=16$ are separated by a vertical dashed line.

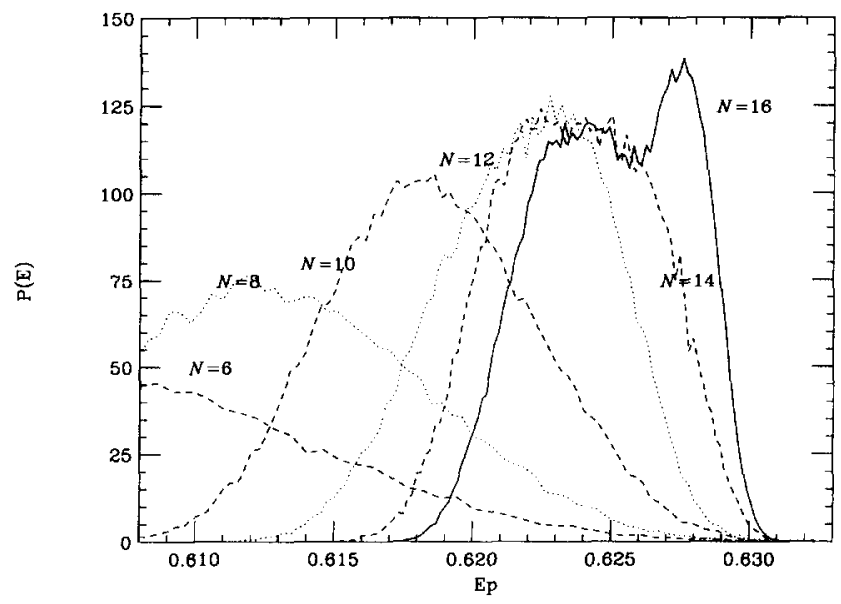

Fig. 18. $E_{\mathrm{p}}$ distributions at $\gamma=-0.2$ on the HS topology.

the value in $N=14$ preempting the extrapolation to $2 / 3$. Unfortunately lattices larger than $N=16$ are unaccessible to our computers nowadays, and we cannot observe a decreasing $V_{L}^{\min }$ as we did for the other $\gamma$ values. However, from the behavior between $N=14$ and $N=16$ an increasing $V_{L}^{\min }$ for larger spheres seems to be very unlikely.

Concerning the effective critical exponent $\nu$ we have measured the position of the first Fisher zero (see Table 1) and compute $\nu_{\text {eff }}$ (see Fig. 11). Again the first-order value $1 / d$ is reached in the largest lattices. 


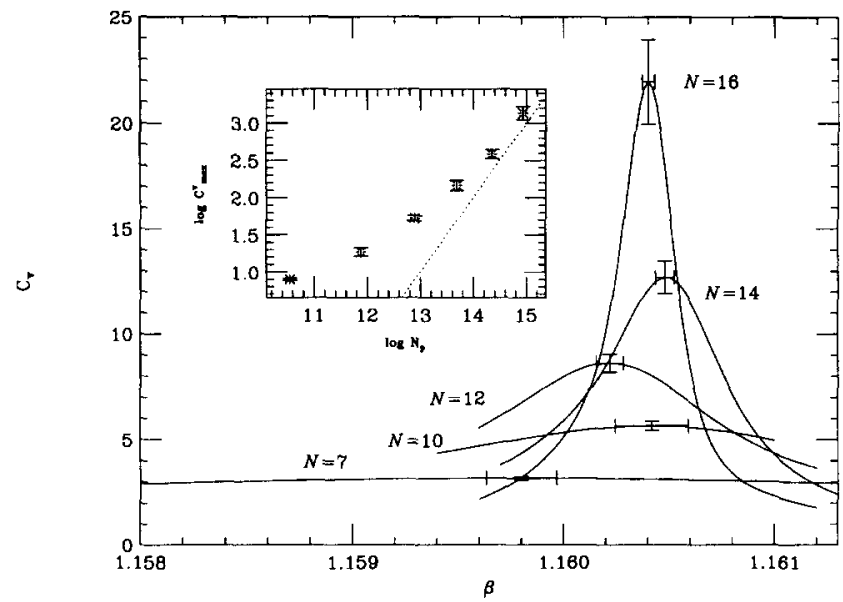

Fig. 19. Specific heat maximum, and Ferrenberg-Swendsen extrapolation (solid line) at $\gamma=-0.2$. The small window represents $C_{\mathrm{v}}^{\max }\left(N_{\mathrm{p}}\right)$ The dotted line correspond to the behavior expected in a first-order phase transition.

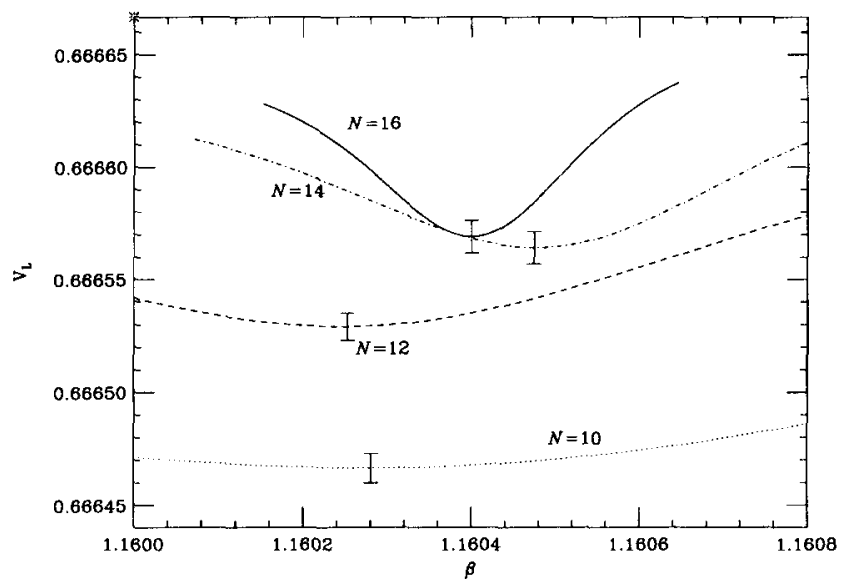

Fig. 20. Binder cumulant at $\gamma=-0.2$ on the HS topology in $N=10,12,14,16$. The cross in the upper corner signals the second-order value $2 / 3$.

\subsection{Toroidal versus spherical topology}

Due to the fact that on the sphere there are a number of points with less than the maximum connectivity, violations to standard FSS in the form of uncontrolled finite size effects are expected to appear on this topology.

We have observed all along this work that working on the spherical topology retards the onset of two-state signals.

In terms of $L_{\text {eff }}$, some qualitative prediction arising from our results would be that at fixed $\gamma$, the minimum lattice size required to observe a double peak structure, $L_{\min }$, is around three times larger on the sphere than on the torus. This one being the showier 


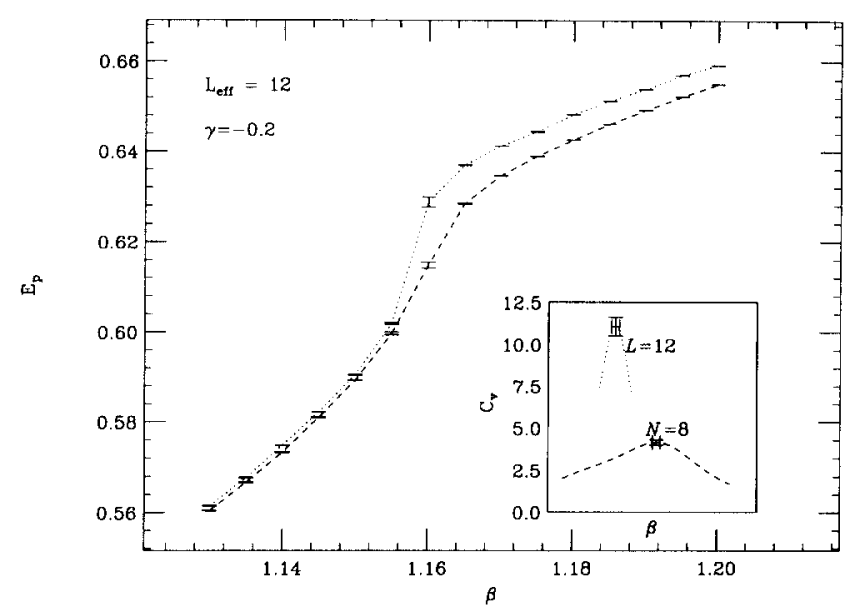

Fig. 21. $E_{\mathrm{p}}$ at $\gamma=-0.2$ in $L_{\mathrm{eff}} \sim 12$. The dotted line corresponds to the toroidal topology, the dashed one to the sphere $N=8$. The small window shows the difference in the specific heat between both cases.

difference, it is certainly not the only one.

When comparing $C_{\mathrm{v}}^{\max }\left(L_{\mathrm{eff}}\right)$ on both topologies one finds always smaller values on the spherical topology ( see small window in Fig. 21). We did run on $L=12$ on the torus and $N=8$ on the sphere, which has an $L_{\text {eff }} \sim 12$, sweeping an interval of $\beta$ values including the phase transition. In Fig. 21 we have plotted $E_{\mathrm{p}}$ in both topologies. In the region of low $\beta$ the system is disordered, the entropy is higher and the system is not so sensitive to inhomogeneities of the lattice. However, in the region of high $\beta$ the energy is in general smaller than for the homogeneous lattice since the system tends to be ordered, and the influence of the sites with less than maximum connectivity is more evident. This difference gets smaller when the lattice size is increased on the sphere, and hence the energy jump is larger. This feature explains the observed splitting of the two states on the spherical lattices when increasing lattice size.

Altogether, it is not recommended to work on spherical lattices to check the existence of two-state signals. However, there are a number of facts that make this topology not so disappointing in spite of those uncontrolled finite size effects. The first one concerns the behavior of $\beta^{*}(L)$ and the second one the measure of the latent heat.

In Fig. 22 we compare $\beta^{*}(L)$ on the torus and on the sphere. A first observation is that despite they not having the same values in finite lattices, both curves get closer when increasing the lattice size. This supports the idea of a common thermodynamic limit for both topologies. On the other hand, the shift in the apparent critical coupling with $L$ is much less dramatic on the sphere than on the torus. It seems that finite size corrections to $\beta_{\mathrm{c}}$ are smaller in the case of the spherical topology. This behavior has also been observed in the Ising model on spherical lattices [31].

The behavior of the latent heat is very significant. In Fig. 23 we compare the latent heat in finite volumes for both topologies. On the Wilson line, at $\gamma=0$ the asymptotic value of the latent heat is obtained in the spherical topology. The inhomogeneity of 


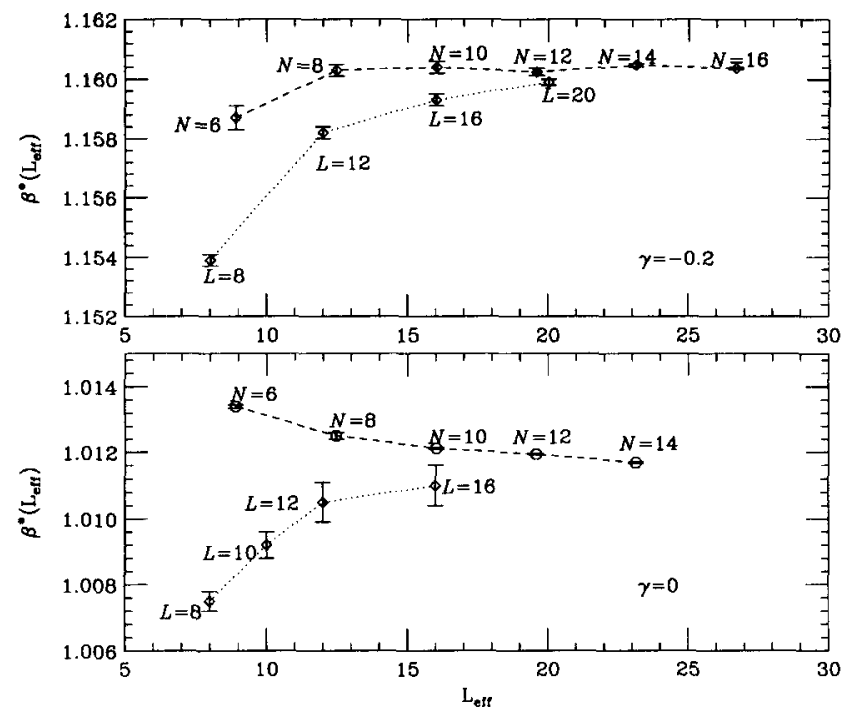

Fig. 22. $\beta^{*}(L)$ for the different lattice sizes on the torus (dotted lines) and on the sphere (dashed lines) at $\gamma=0$ (lower window) and at $\gamma=-0.2$ (upper window). The couplings for $\gamma=0$ on the torus have been taken from $[10]$.

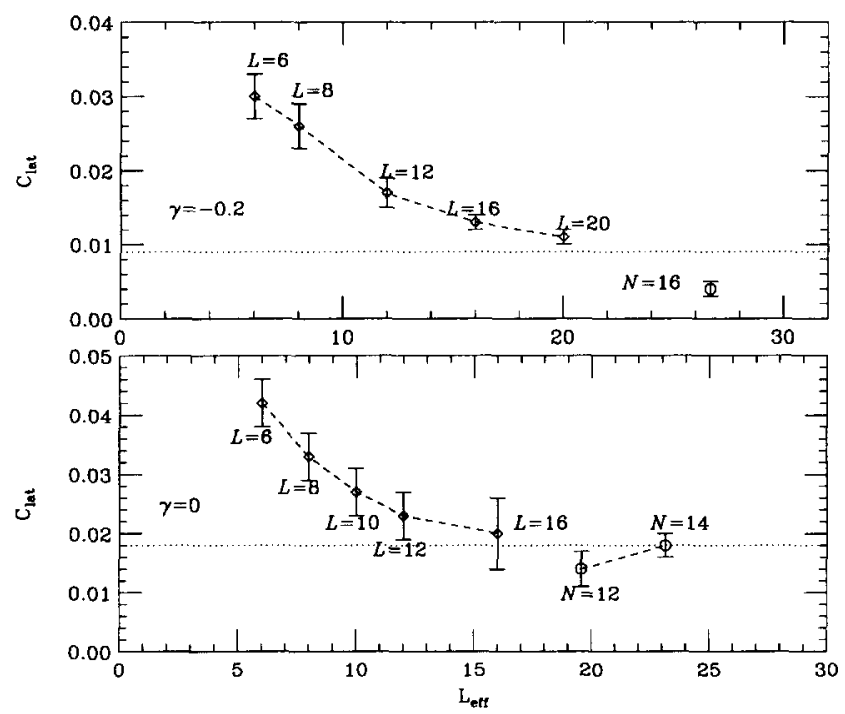

Fig. 23. Latent heat for the different lattice sizes on the torus and on the sphere at $\gamma=0$ (lower window) and at $\gamma=-0.2$ (upper window). The dotted line corresponds to $C_{\text {lat }}(\infty)$ obtained by extrapolating the values on the torus through a linear fit as function of $1 / V$. The values for $\gamma=0$ on the torus have been taken from $\{10\rceil$. 
the sphere has, paradoxically, helped us to run lattices with $L_{\text {eff }} \sim 24$ without having to worry about the tunneling rate. From this graph we quote $C_{\text {lat }}(\infty)=0.018(1)$. In the upper window the same is plotted for $\gamma=-0.2$. Unfortunately we just have a single lattice size on the sphere to measure the latent heat, however, from the behavior exhibited by this topology, an increase of the latent heat on spheres larger than $N=16$ seems rather likely. Altogether, at $\gamma=-0.2$ a $C_{\text {lat }}(\infty) \sim 0.009$ is plausible.

In what concerns the latent heat, the spherical topology seems to afford an useful complement to the results obtained for the toroidal lattice. In fact, on the Wilson line lattices larger than $L=16$ cannot be studied due to the technical problem associated to the scarce tunneling. The spherical lattice alleviates this technical problem, and the results are supporting the value of the extrapolated latent heat as a function of the inverse of the volume, from the data on toroidal lattices up to $L=16$. One could consider the possibility of simulating on spherical lattices at negative $\gamma$ to solve the tunneling problem, but in our opinion, the price to pay is too high.

\section{Conclusions}

The first-order character of the deconfinement transition in pure $U(1)$ has been proved, up to the limits of a rather reasonable numerical evidence, in the interval $\gamma \in[+0.2,-0.4]$.

In $\gamma=+0.2$ we have been able to stabilize the latent heat. We are aware of no simulation on the torus showing a stable latent heat due to the scarce tunneling in lattices larger than $L=6$. The spherical topology has helped to solve this problem. However, probably any lattice with inhomogeneities would produce the same catalyzing effects.

In $\gamma=0$ we have also been able to measure the asymptotic value of the latent heat. We have proved the suggestions of several authors about a quasi-stabilization on lattices larger than $L \sim 12$. The data on the spherical topology have been crucial to discard the possibility of a slowly vanishing latent heat. It follows that the discretization of pure compact U(1) LGT on the lattice using the Wilson action exhibits a first-order phase transition with a latent heat in the thermodynamical limit $C_{\text {lat }} \sim 0.018$.

On the toroidal topology things happen qualitatively in the same way than in $\gamma=0$ up to $\gamma=-0.4$. We have run on spherical lattices in $\gamma=-0.2$ looking for an argument similar to the one found in the Wilson case concerning the stabilization of the latent heat. The simulation in $N=16$ gives an estimation for the latent heat though rather imprecise because the splitting of the peaks is not good enough for the measurement to be accurate. However, in view of the behavior exhibited by the spherical topology concerning the trend of the two peaks on spherical lattices to separate, we are prone to consider the value measured in $N=16$ as a lower bound for the latent heat in $\gamma=-0.2$. On the other hand, we would at present say that the latent heat extrapolated from the data on the torus up to $L=20$ is rather accurate, in view of the behavior for the Wilson case. The possibility of running larger spheres surpasses our computer resources. A 
highly parallelized version of the code should be used to alleviate thermalization, which is a possibility we do not discard completely at medium term.

Either proving or discarding the possibility of a TCP at some finite negative $\left|\gamma_{\mathrm{TCP}}\right|>$ 0.4 will be a very difficult task from the numerical point of view. An analytical argument would be welcome. A very tiny two-state signal, comparable with the one observed in $L=8$ at $\gamma=-0.4$, is observed on the torus in $L=16$ at $\gamma=-0.8$, which is the most negative value we have run on the torus. Taking into account the factor 3 in $L_{\text {eff }}$ one would need an $N \sim 30$ sphere to observe a tiny double peak structure. Simulation with spherical lattices in this range of $\gamma$ have no sense, and nothing can be concluded from the absence of two-state signals from such negative $\gamma$ values.

In view of these difficulties to stabilize the latent heat for very negative $\gamma$ values, the only chance to discern the order of the phase transition is the study of effective critical exponents. Cumulants of the energy, such as the Binder cumulant, have been shown to behave as expected in the first-order case only when the stable two-peak structure is almost setting in, and there, we do not need further evidences any longer. We could not expect additional information since by definition the Binder cumulant relies on the existence of stable latent heat in order to extrapolate to $V_{L=\infty}^{\min }<2 / 3$.

The advantage of studying the effective exponents (which is nothing but studying the evolution of the histograms width) is that we do not need a direct observation of latent heat to conclude that a transition is first order [36,33]. We have observed a $\nu_{\text {eff }}$ which evolves monotonically until it reaches the first-order value, namely 0.25 , in all cases. The statistics needed to observe a monotonous behavior, are order $1000 \tau$ at the coupling $\beta^{*}(L)$, which has to be located with high precision (four digits in our experience) in order to accurately measure effective exponents. However, one has to make every effort to observe the trend of the effective exponents, since it is the only chance to discern the order of such tricky transitions.

In pure $U(1)$, for the Wilson case, an exponent $\nu \sim 1 / 3$ was widely predicted [2,3,11] at the beginning of the eighties when the lattice sizes where too small to reveal two peaks. That $\nu$ was shown to become $\nu \sim 0.29$ when simulating $L=14$ [30]. We have measured $\nu=0.25$ on spherical lattices and stated its first-order character. Within the approximation of effective potentials it can be shown that along the transient region of a weak first-order phase transition, everything goes like in a second-order one with a thermal exponent $\alpha=0.5$ [20]. Together with Josephson law $(\alpha=2-\nu d)$ it implies $\nu \sim 0.37$. This statement has been checked in 2D Potts [20], 3D and 4D $O(N)$ models [32-35] and in the 4D SU(2)-Higgs at $T=0$ with fixed Higgs modulus [36]. Our results prove that pure compact $U(1)$ theory behaves in the same way.

A general remark to draw from this paper is though that there is no theorem constraining transitions presenting $\nu_{\text {eff }} \sim 1 / 3$ to be of WFO, yet the occurrence of such an exponent is a warning sign to consider the phase transition as suspicious of being first order. 


\section{Appendix A. Schwinger-Dyson equations}

As a further check we have implemented the Schwinger-Dyson equations (SDE) [28] which allow one to recover the simulated couplings from the Monte Carlo data.

Let $A(\theta)$ be an operator with null expectation value,

$$
\langle A(\theta)\rangle=Z^{-1} \int[d \theta] A(\theta) e^{-S \mid \theta\rfloor} \equiv 0 .
$$

Deriving with respect to $\theta$ this equation trivially yields

$$
\left\langle\frac{\partial A(\theta)}{\partial \theta}\right\rangle=\left\langle A(\theta) \frac{\partial S[\theta]}{\partial \theta}\right\rangle \text {. }
$$

which is the equation of motion of the operator $\mathrm{A}(\theta)$ or Schwinger-Dyson equation. When the action depends on several couplings Eq. (A.2) can be expressed as

$$
\left\langle\frac{\partial A(\theta)}{\partial \theta}\right\rangle=\sum_{i} \beta_{i}\left\langle A(\theta) \frac{\partial S_{i}[\theta]}{\partial \theta}\right\rangle .
$$

This equation relates the values of the couplings with the expectation values we measure from the MC simulation. We need as many independent equations as couplings we have to determine in the action. In our case, in order to measure both $\beta$ and $\gamma$, we need two operators with null expectation value in order to have two independent tests. At each lattice site $n$ and for every direction $\mu$ we consider the operators

$$
\begin{aligned}
& A(\theta)=\sin \theta_{p}=\sin \left(\theta_{n, \mu}-\theta_{\text {stap }}\right), \\
& B(\theta)=\sin 2 \theta_{p}=\sin 2\left(\theta_{n, \mu}-\theta_{\text {stap }}\right),
\end{aligned}
$$

where $\theta_{\text {stap }}$ is the staple of the link labeled by $(n, \mu)$.

Applying Eq. (A.3) to those operators we get

$$
\begin{aligned}
\left\langle\sum_{\mathrm{p}} \cos \left(\theta_{n, \mu}-\theta_{\text {stap }}\right)\right\rangle= & \beta\left\langle\sum_{\mathrm{p}} \sin \left(\theta_{n, \mu}-\theta_{\text {stap }}\right) \sum_{\mathrm{p}} \sin \left(\theta_{n, \mu}-\theta_{\text {stap }}\right)\right\rangle \\
& +2 \gamma\left\langle\sum_{\mathrm{p}} \sin \left(\theta_{n, \mu}-\theta_{\text {stap }}\right) \sum_{\mathrm{p}} \sin 2\left(\theta_{n, \mu}-\theta_{\text {stap }}\right)\right\rangle \\
\left\langle\sum_{\mathrm{p}} 2 \cos 2\left(\theta_{n, \mu}-\theta_{\text {stap }}\right)\right\rangle= & \beta\left\langle\sum_{\mathrm{p}} \sin 2\left(\theta_{n, \mu}-\theta_{\text {stap }}\right) \sum_{\mathrm{p}} \sin \left(\theta_{n, \mu}-\theta_{\text {stap }}\right)\right\rangle \\
& +2 \gamma\left\langle\sum_{\mathrm{p}} \sin 2\left(\theta_{n, \mu}-\theta_{\text {stap }}\right) \sum_{\mathrm{p}} \sin 2\left(\theta_{n, \mu}-\theta_{\text {stap }}\right)\right\rangle
\end{aligned}
$$

On the hypertorus $\sum_{p}$ means the sum over the plaquettes in positive and negative directions $( \pm \mu)$ bordering the link (12 plaquettes). On the sphere one has to be 
Table A.1

Couplings obtained from the MC simulations at $\gamma=-0.2$ using the Schwinger-Dyson equations

\begin{tabular}{rllllll}
\hline \multicolumn{1}{r}{$L$} & \multicolumn{2}{c}{ HT topology } & & \multicolumn{2}{c}{ HS topology } \\
\cline { 2 - 3 } \cline { 5 - 6 } & $\beta_{\text {sim }}$ & $(\beta, \gamma)_{\mathrm{SD}}$ & $N$ & $\beta_{\text {sim }}$ & $(\beta, \gamma)_{\mathrm{SD}}$ \\
\hline 6 & 1.1460 & $1.1466(44),-0.2009(26)$ & 6 & 1.1587 & $1.1592(14),-0.1998(12)$ \\
8 & 1.1535 & $1.1527(22)-0.1997(15)$ & 8 & 1.1600 & $1.1599(12)-0.1999(8)$ \\
12 & 1.1582 & $1.1581(12),-0.1999(8)$ & 10 & 1.1602 & $1.1602(7),-0.2001(9)$ \\
\hline
\end{tabular}

careful, since not all the links have 12 surrounding plaquettes, and the sum has to be understood as extended to the existing plaquettes.

These equations hold for all $n$ in such a way that we can sum up the equations for every single link and average over the number of links. We quote in Table A.1 for the tests done at $\gamma=-0.2$ using both topologies. They show a perfect agreement between the simulated couplings and the ones recovered from the $\mathrm{MC}$ expectation values.

\section{Acknowledgements}

We have benefited from comments and discussions with L.A. Fernández and J. Salas. The discussions with C.B. Lang and J. Jersák at the final stage of the work have been very appreciated by the authors.

I.C. feels indebted to J. Jersák for his many stimulating comments as well as to C.B. Lang for his warm hospitality.

The simulations have been done using the RTNN machine at Theoretical Physics Department, University of Zaragoza (Spain). I.C. is a MEC fellow.

\section{References}

[1] M. Creutz, L. Jacobs and C. Rebbi, Phys. Rev. D 20 (1979) 1915.

[2] B. Lautrup and M. Nauenberg, Phys. Lett. B 95 (1980) 63.

[3| G. Bhanot, Phys. Rev. D 24 (1981) 461.

[4] J. Jersák, T. Neuhaus and P.M. Zerwas, Phys. Lett. B 133 (1983) 103.

15] V. Azcoiti, G. di Carlo and A. Grillo, Phys. Lett. B 238 (1990) 355.

[6] G. Bhanot, T. Lippert, K. Schilling and P. Ueberholz, Nucl. Phys. B 378 (1992) 633.

[7] C.B. Lang and C. Rebbi, Phys. Rev. D 35 (1987) 2510.

[8] D.J. Callaway and R. Petronzio, Nucl. Phys. B 280 (1987) 481.

[9] P. Hasenfratz and A. Hasenfratz, Nucl. Phys. B 295 (1988) 1.

[10] L.A. Fernández, A. Muñoz-Sudupe, R. Petronzio and A. Tarancón. Phys. Lett. B 267 (1991) 100.

[11] G. Bhanot, Nucl. Phys. B 205 (1982) 168.

[12] H.G. Evertz, J. Jersák, T. Neuhaus and P.M. Zerwas, Nucl. Phys. B 251 (1985) 279.

[13| A. Hasenfratz, Phys. Lett. B 201 (1988) 492.

[14] R. Gupta, M.A. Novotny and R. Cordery, Phys. Lett. B 172 (1986) 86.

[15] C.B. Lang and T. Neuhaus, Nucl. Phys. B 431 (1994) 119.

[16] M. Baig and H. Fort, Phys. Lett. B 332 (1994) 428.

[17] J. Jersák, C.B. Lang and T. Neuhaus, Phys. Rev. Lett. 77 (1996) 1933.

[18] W. Kerler, C. Rebbi and A. Weber, Phys. Lett. B 348 (1995) 565. 
| $19 \mid$ J. Cox, W. Franzki, J. Jersák, C.B. Lang and T. Neuhaus, (preprint hep-lat://9705043).

[20] L.A. Fernández, M.P. Lombardo, J.J. Ruiz-Lorenzo and A. Tarancón, Phys. Lett. B 277 (1992) 485.

[21] The APE collaboration, Nucl. Phys. B 318 (1989) 553.

[22] W. Kerler, C. Rebbi and A. Weber, Nucl. Phys. B 452 (1995) 452.

[23] I. Campos, A. Cruz and A. Tarancón, To appear in Phys. Lett. B.

[24] J. Lee and J.M. Kosterlitz, Phys. Rev. Lett. 65 (1990) 137.

|25] M.S. Challa, D.P. Landau, K. Binder, Phys. Rev. D 34 (1986) 1841.

[26] C.N. Yang and T.D. Lee. Phys. Rev. 87 (1952) 404.

|27| E. Marinari, in 1996 Budapest Summer School on Monte Carlo methods e-print: cond-mat//9612010.

[28] M. Falcioni, G. Martinelli, M.L. Paciello, G. Parisi and B. Taglienti, Nucl. Phys. B 265 (1986) 187.

[29] A.D. Sokal, Bosonic Algorithms, in Quantum Fields on the Computer. Advanced Series on Direction in High Energy Physics, Vol. 11, ed. M. Creutz (World Scientific, Singapore 1992).

[30| V. Azcoiti, G. di Carlo and A. Grillo, Phys. Lett. B 268 (1991) 101.

[31] O. Diego, J. Gonzalez and J. Salas, J. Phys. A 27 (1994) 2965.

[32| J.L. Alonso, J.M. Carmona, J. Clemente, L.A. Fernández, D. Iñiguez, A. Tarancón and C.L. Ullod, Phys. Lett. B 376 (1996) 148.

|33| H.G. Ballesteros, J.M. Carmona, L.A. Fernández and A. Tarancón, Phys. Lett. B 419 (1998) p. 303.

[34] I. Campos, L.A. Fernández and A. Tarancón, Phys. Rev. D 55 (1997) 2965.

135| J.L. Alonso, H.G. Ballesteros, L.A. Fernández, V. Martín-Mayor, A. Muñoz Sudupe and A. Tarancón, Phys. Rev. B 53 (1996) 2537.

[36] I. Campos, Nucl. Phys. B 514 (1998) 336. 